\title{
Overview of the LHCb Calorimeter System
}

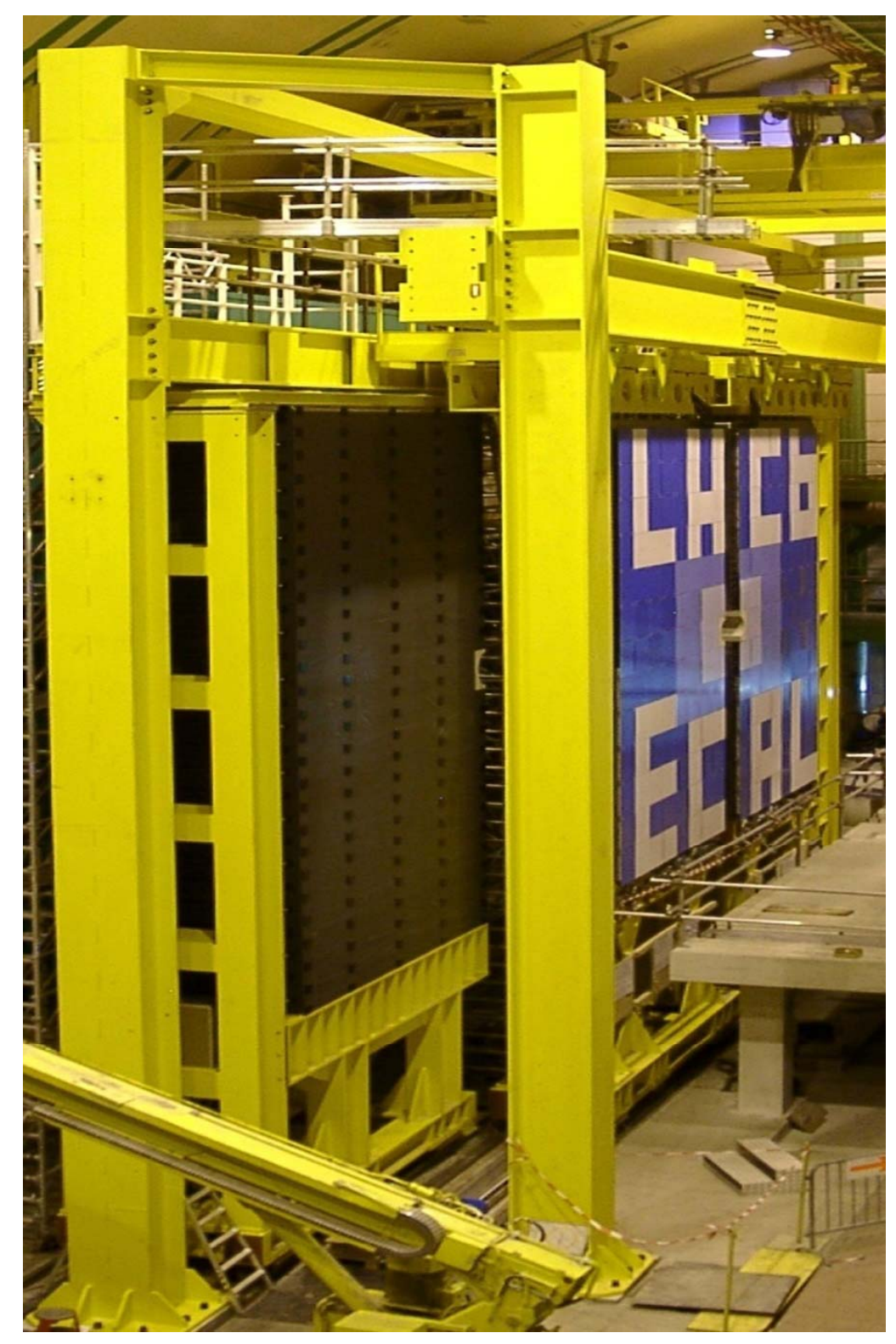

\begin{tabular}{|c|}
\hline TIPP09 \\
Tsukuba, Japan \\
12-17 March 2009 \\
\hline
\end{tabular}

Outline:

$>$ Overview of $\mathrm{LHCb} \&$ requirements to the calorimeter system

$>$ Design, construction \& performance of the sub-systems

$>$ Conclusion

$$
\begin{gathered}
\text { presented by } \\
\text { Andreas Schopper (CERN) }
\end{gathered}
$$

on behalf of the LHCb Collaboration 


\section{LHCb Detector Overview}

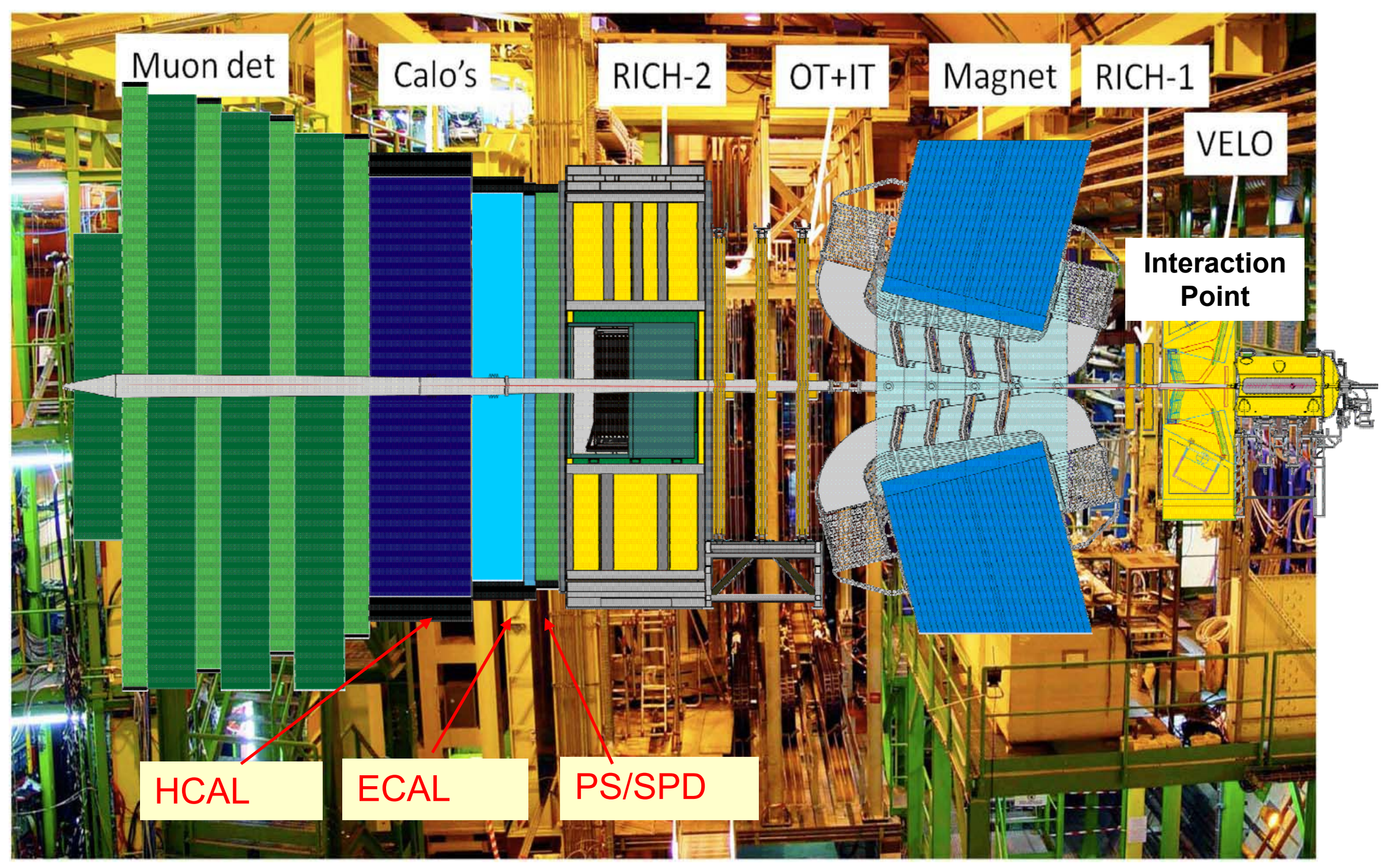

LHCP Tsukuba, March 2009

TIPP09 


\section{Detector requirements}

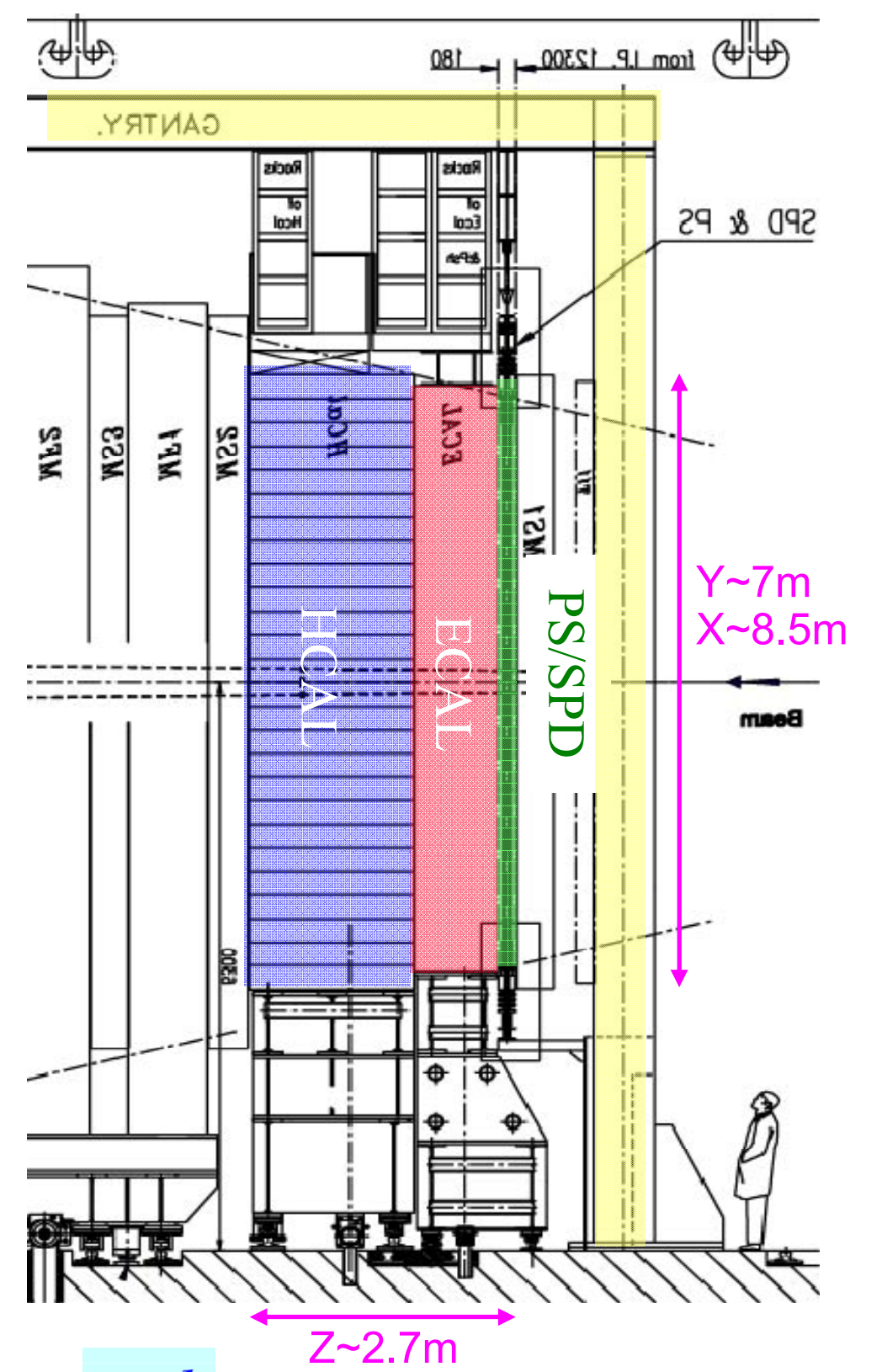

KHCp $\frac{Z \sim 2.7 \mathrm{~m}}{\text { Tsukuba, March } 2009}$ $\underline{\text { Purpose of the LHCb Calorimeter System }}$

Preshower (PS) and

Scintillator Pad Detector (SPD):

- PID for L0 electron and photon trigger

- electron, photon/pion separation by PS

- photon/MIP separation by SPD

- charged multiplicity veto by SPD

Electromagnetic Calorimeter (ECAL):

- $\mathrm{E}_{\mathrm{t}}$ of electrons, photons and $\pi^{0}$ for L0 trigger (e.g. B $\rightarrow J / \Psi \mathrm{Ks}, \mathrm{B} \rightarrow \mathrm{K}^{*} \gamma$ )

- reconstruction of $\pi^{0}$ and prompt $\gamma$ offline

- particle ID

Hadron Calorimeter (HCAL):

- $\mathrm{E}_{\mathrm{t}}$ of hadrons for L0 trigger

(e.g. $\mathrm{B} \rightarrow \pi \pi, \mathrm{B} \rightarrow \mathrm{D}_{\mathrm{s}} \mathrm{K}$ )

- particle ID

L0 trigger $=>$ Calorimeters readout every $25 \mathrm{~ns}$ 


\section{Level-0 calorimeter trigger}

$>$ Measure energy and identify $\gamma / \mathrm{e} / \mathrm{h}$ using SPD/PS/ECAL/HCAL in coincidence

$>$ SPD \& PS validate the charged and EM nature of incoming particle, respectively

$\rightarrow$ Used at the fast Level-0 trigger

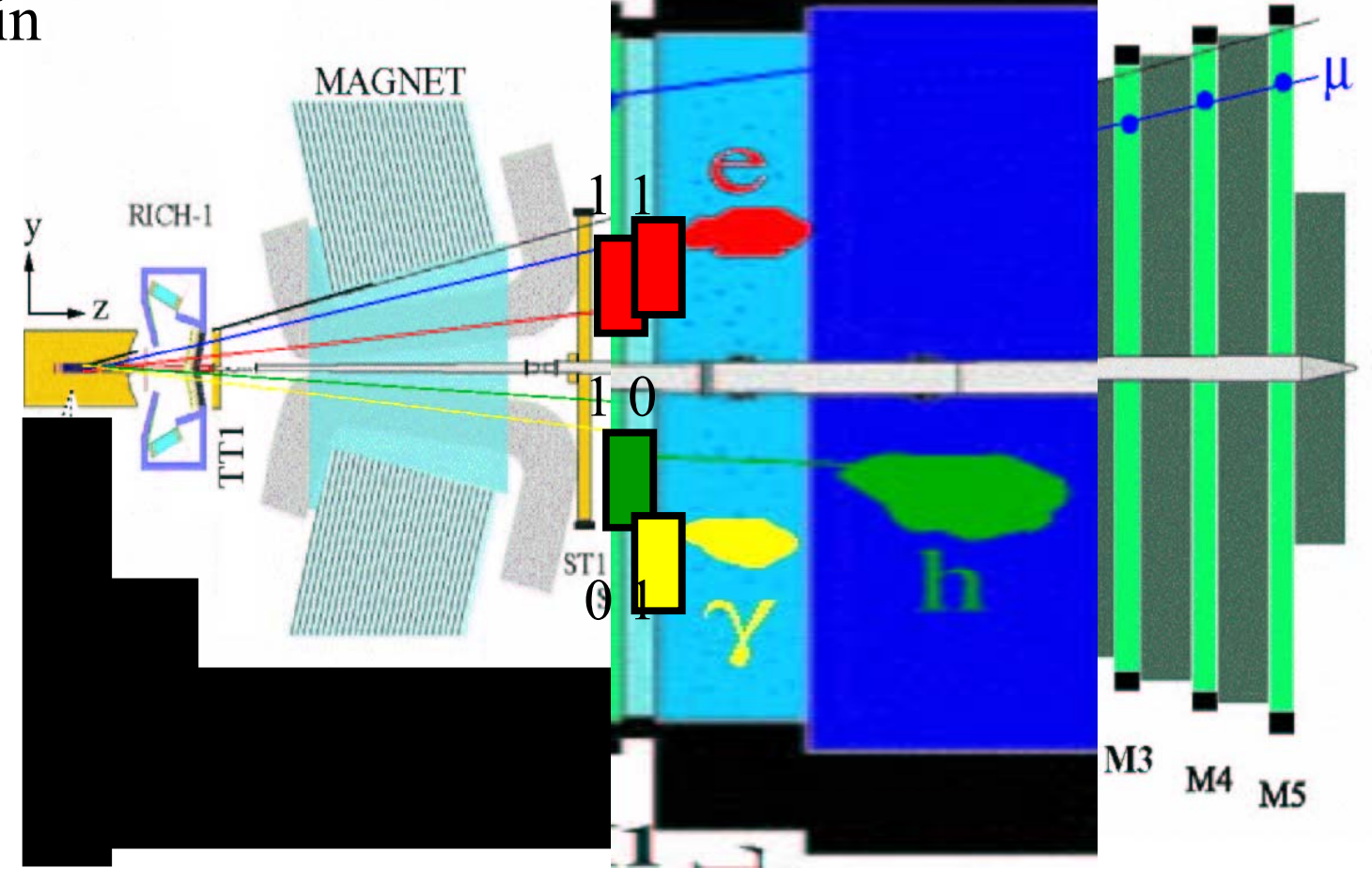

$\checkmark 40 \mathrm{MHz}$ hardware trigger.

$\checkmark$ identify high $\mathrm{P}_{\mathrm{t}}$ particles that could sign a B decay

$\checkmark$ use Calorimeter and Muon sub-systems

$\checkmark \quad 10 \mathrm{MHz}$ visible interactions $\rightarrow 1 \mathrm{MHz}$ 


\section{ECAL \& HCAL choice of technology}

ECAL design requirements:

energy resolution $\sim 10 \% / \sqrt{\mathrm{E}} \oplus 1 \%$

$>$ fast response time compatible with LHC bunch spacing of $25 \mathrm{~ns}$

$>$ radiation hard up to $\sim 250 \mathrm{krad} /$ year

$>$ small transverse segmentation to separate two showers from $\pi^{0}$ decays and to minimize pile-up

$>$ lateral size of active area $7.8 \mathrm{~m} \times 6.3 \mathrm{~m}$

cost effective
HCAL design requirements:

$>$ fast (25 ns cycle)

$>$ moderate resolution is sufficient $\sim 80 \% / \sqrt{\mathrm{E}} \oplus 10 \%$

$>$ radiation tolerance up to $\sim 50 \mathrm{krad} / \mathrm{year}$ in the inner zone

$>$ transverse segmentation to minimize pile-up

$>$ longitudinal depth limitations

$>$ lateral size $8.4 \mathrm{~m} \times 6.8 \mathrm{~m}$

$>$ cost effective

$\oplus$ common readout electronics

$\rightarrow$ sampling technology using scintillators \& fibres with readout by common photomultiplier \& FE electronics

$\rightarrow$ "shashlik" module

$\rightarrow$ "tile-cal" module 


\section{ECAL Shashlik modules}

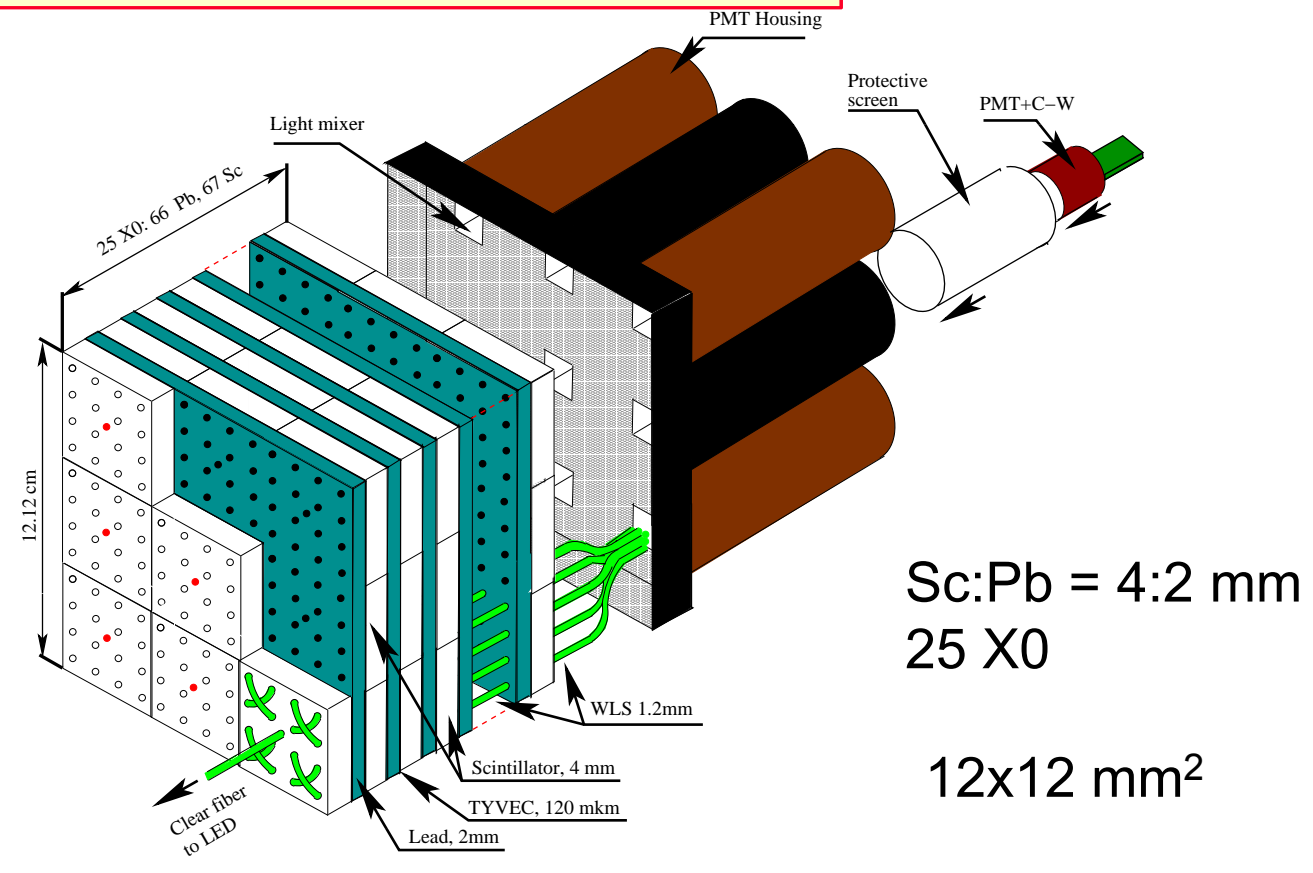

\section{2 shashlik modules with 25 X0 Pb}

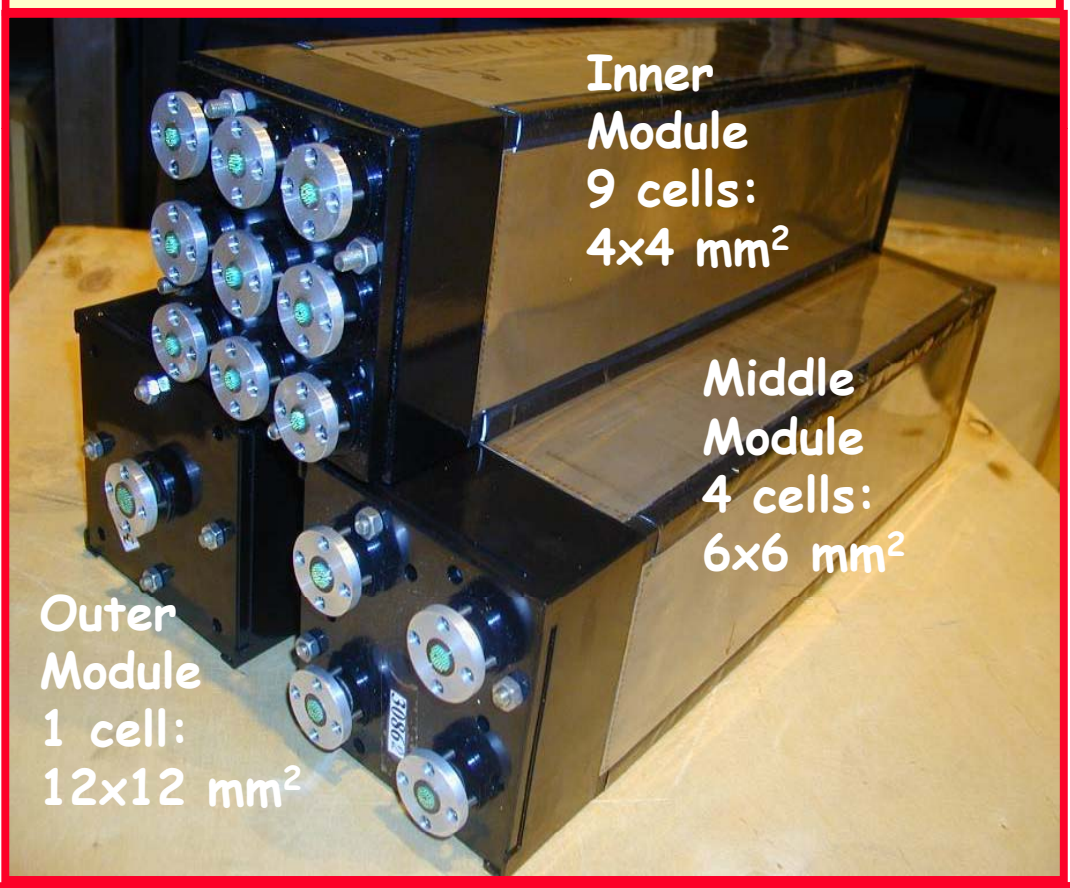

\section{Scintillators, lead-plates, covers}

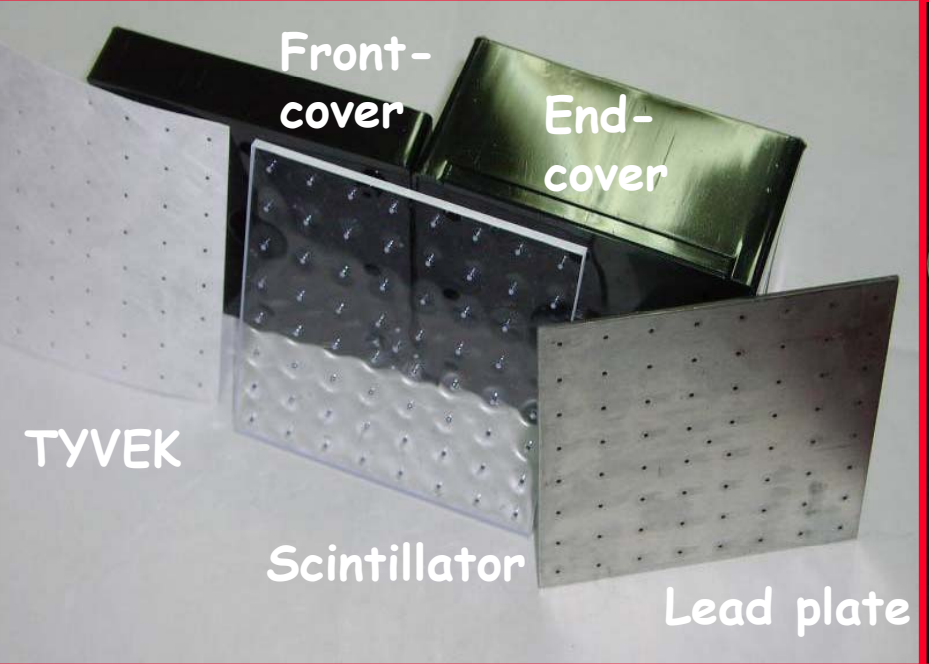

KHCp Tsukuba, March 2009

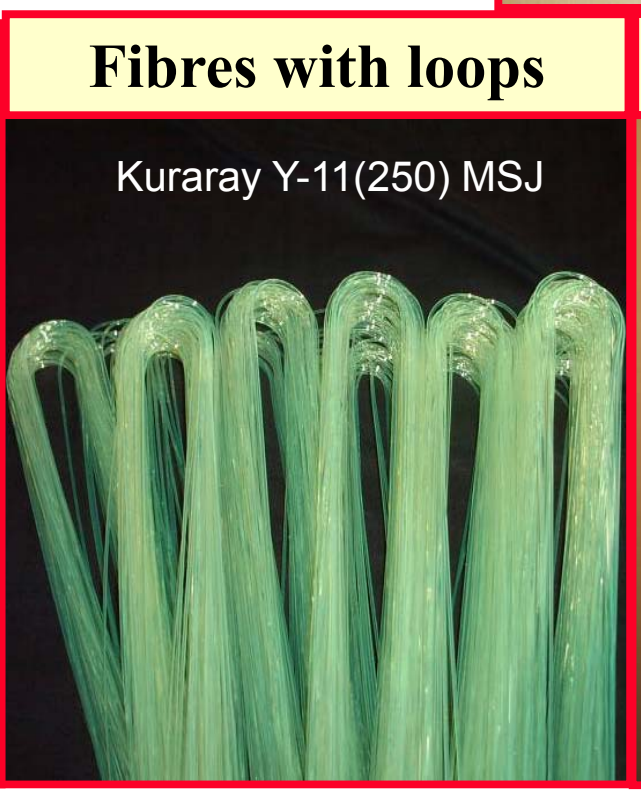

Kuraray Y-11(250) MSJ

TIPP09

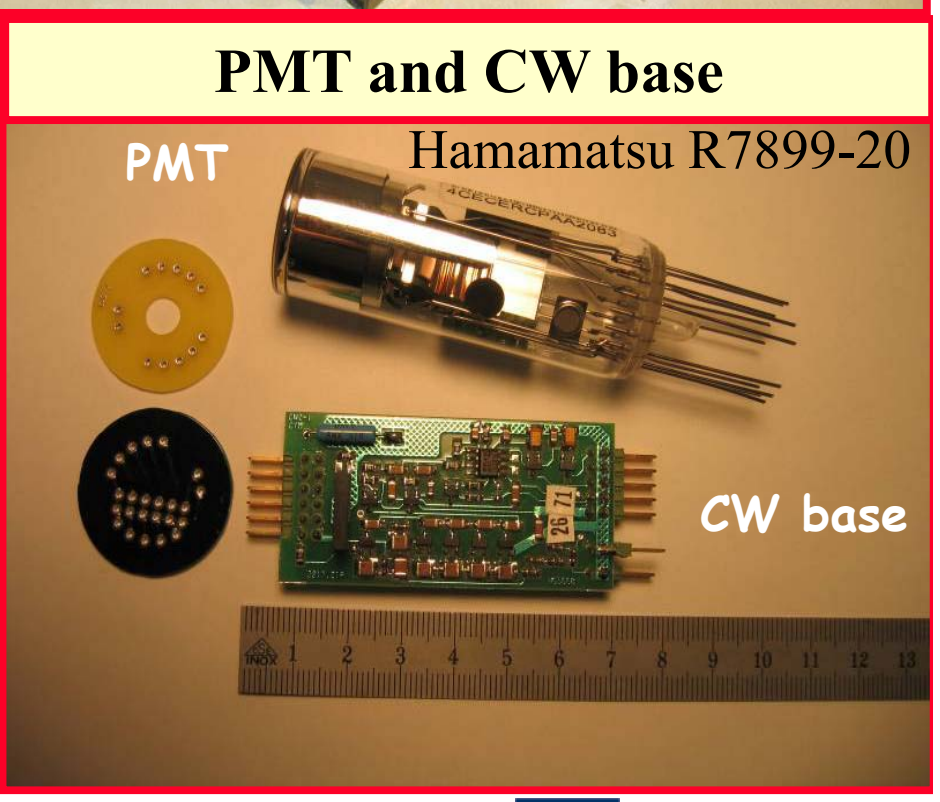




\section{ECAL module performance}

'dead time'-less shaping \& integration of PM signal at $40 \mathrm{MHz}$

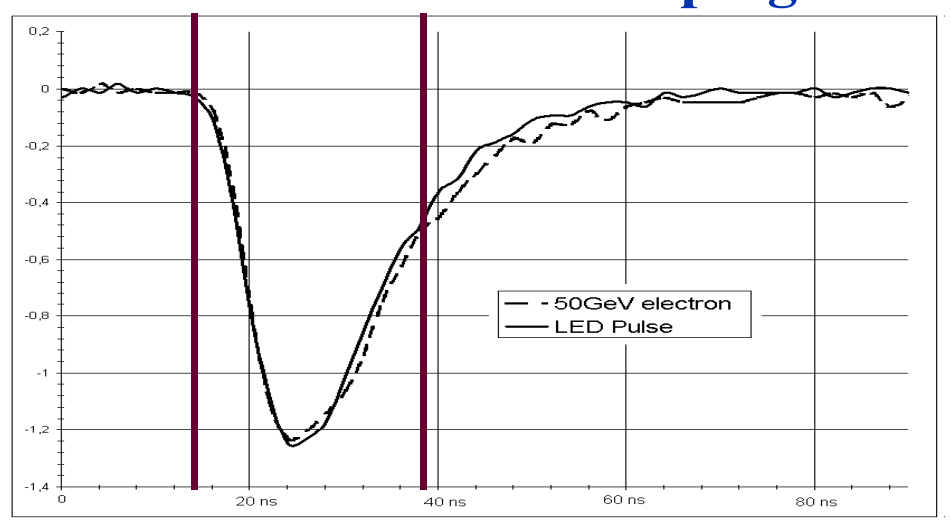

PM anode current pulse

\begin{tabular}{|l|l|l|l|}
\hline & Inner & Middle & Outer \\
\hline $\begin{array}{l}\text { <Light yield> per cell, } \\
\text { Nph.e./GeV }\end{array}$ & 3100 & 3500 & 2600 \\
\hline $\begin{array}{l}\text { MIP response, cell-to-cell } \\
\text { variation, \% }\end{array}$ & $8 \%$ & $5.3 \%$ & $6.7 \%$ \\
\hline
\end{tabular}

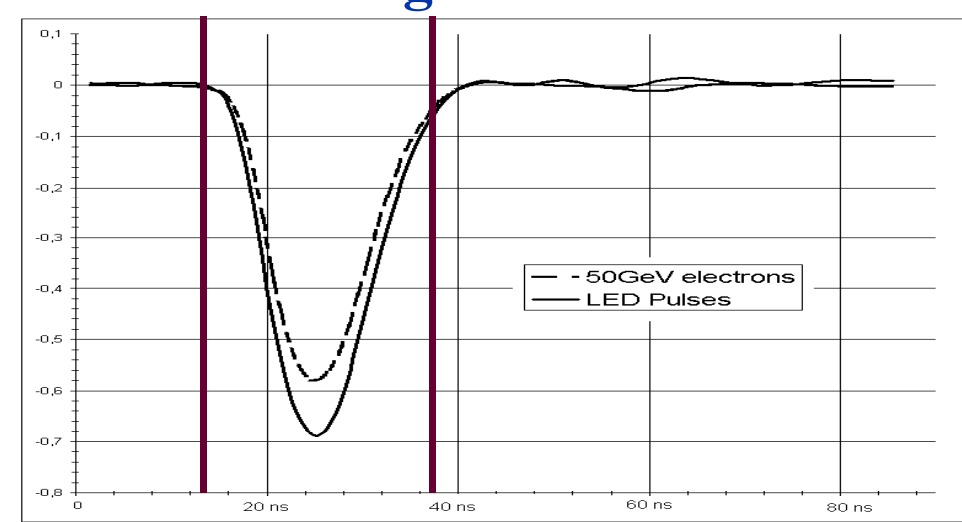

Pulse after clipping chain

Energy resolution with electrons

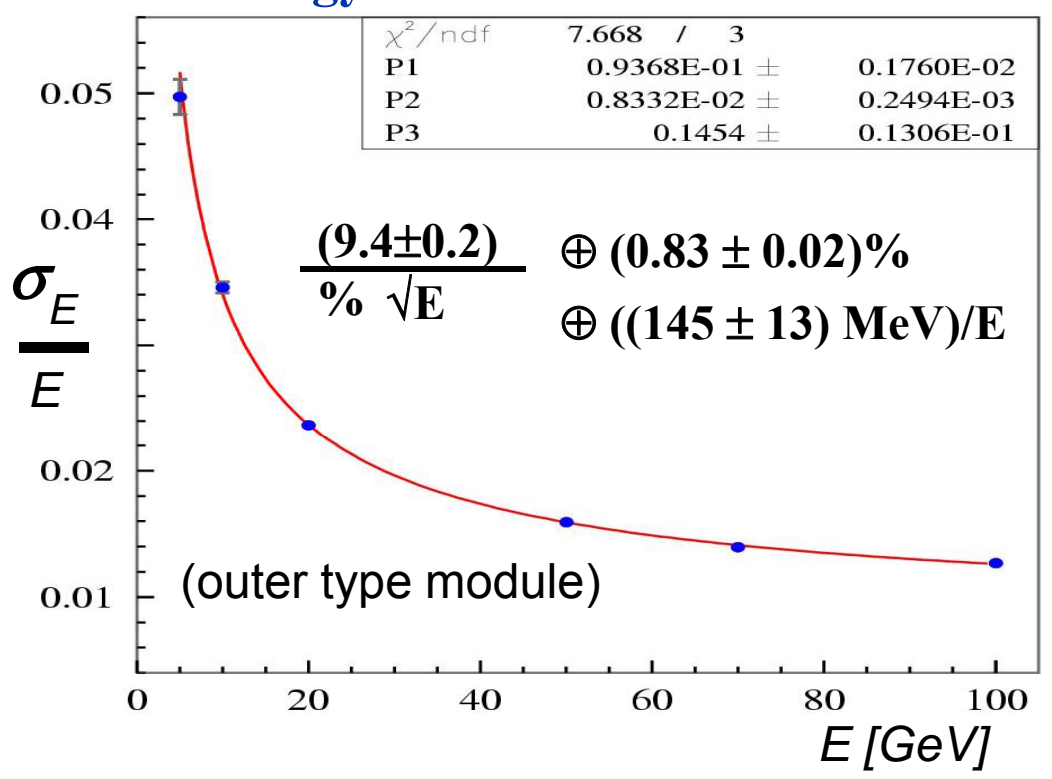




\section{ECAL uniformity of light response}

Transverse scan with $50 \mathrm{GeV}$ electrons

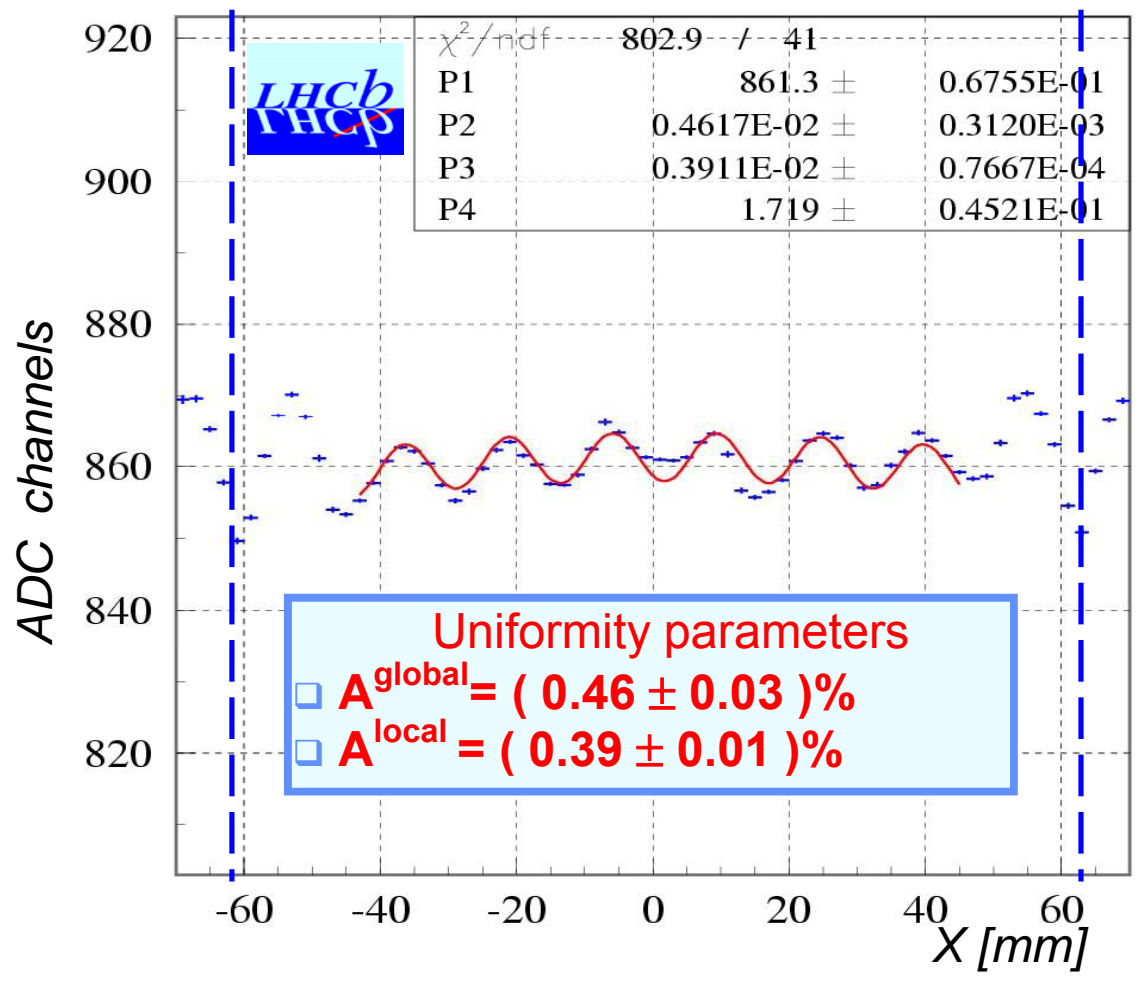

Transverse scan with $80 \mathrm{GeV}$ electrons

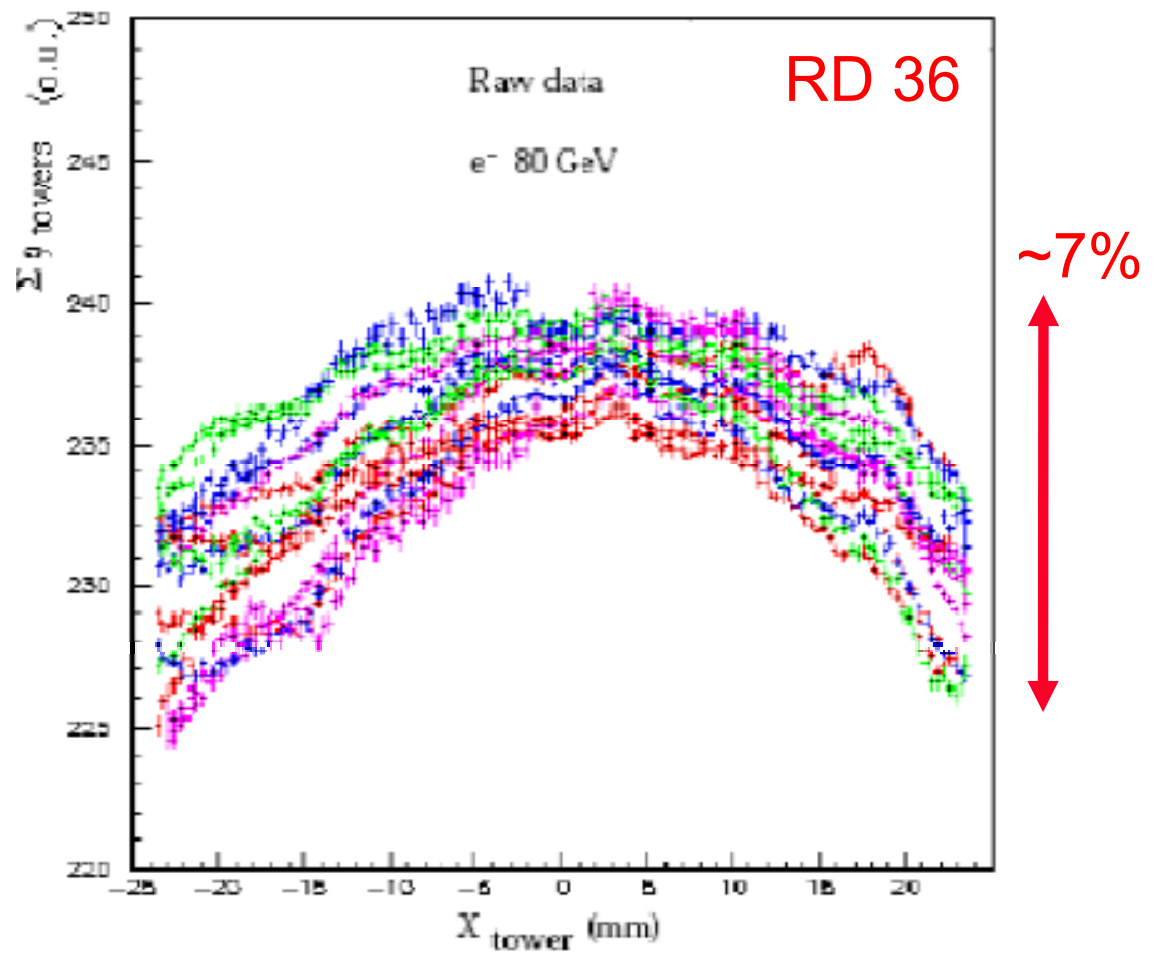




\section{Simulation of response uniformity}

MC modeling:

- light collection efficiency $\rightarrow$ ray tracer program (refraction, reflection, attenuation...)

- Scintillator thickness $\rightarrow$ measured

- Convolution with particle energy deposition $\rightarrow$ GEANT

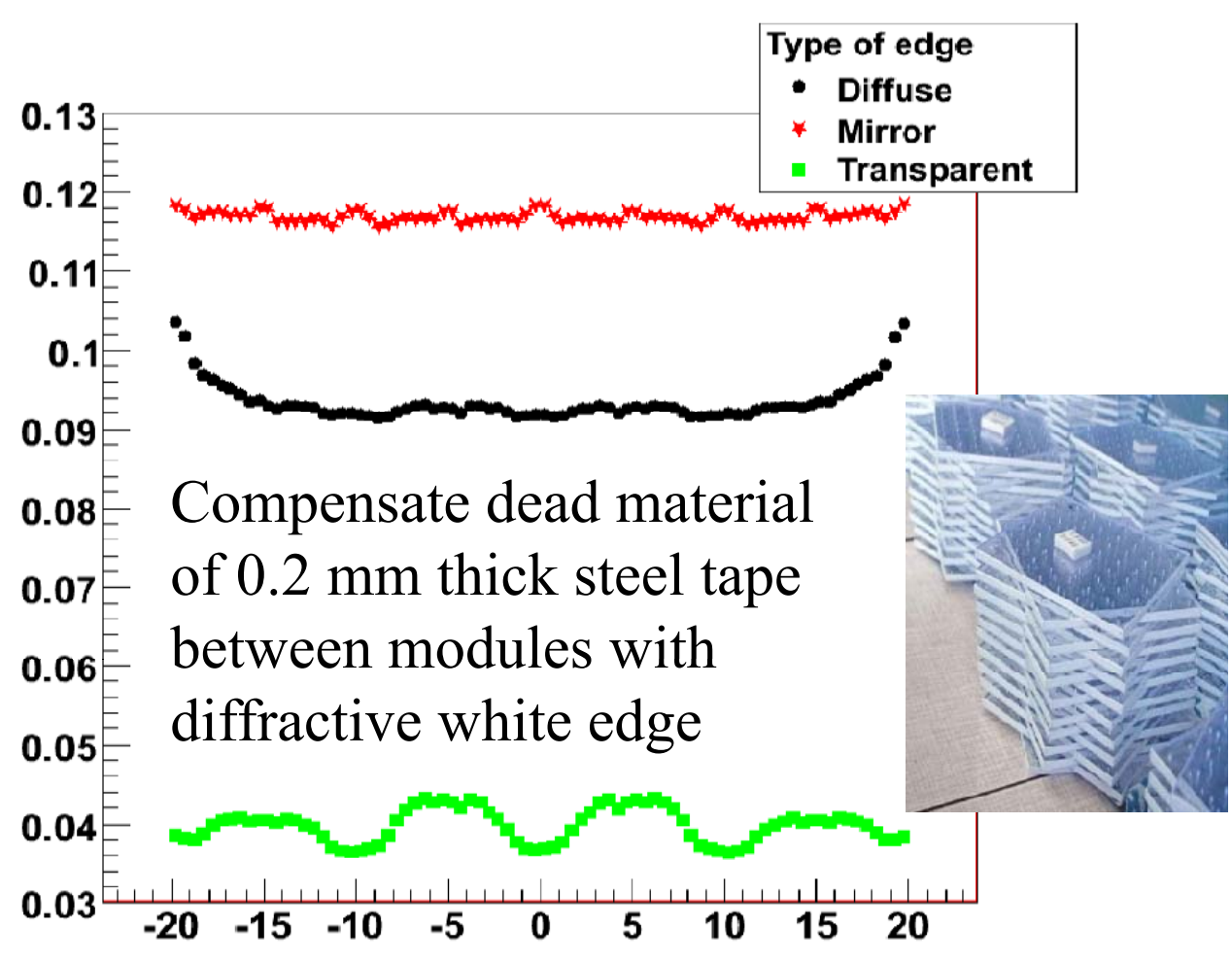

\section{Data (points) vs simulation (grey area)}
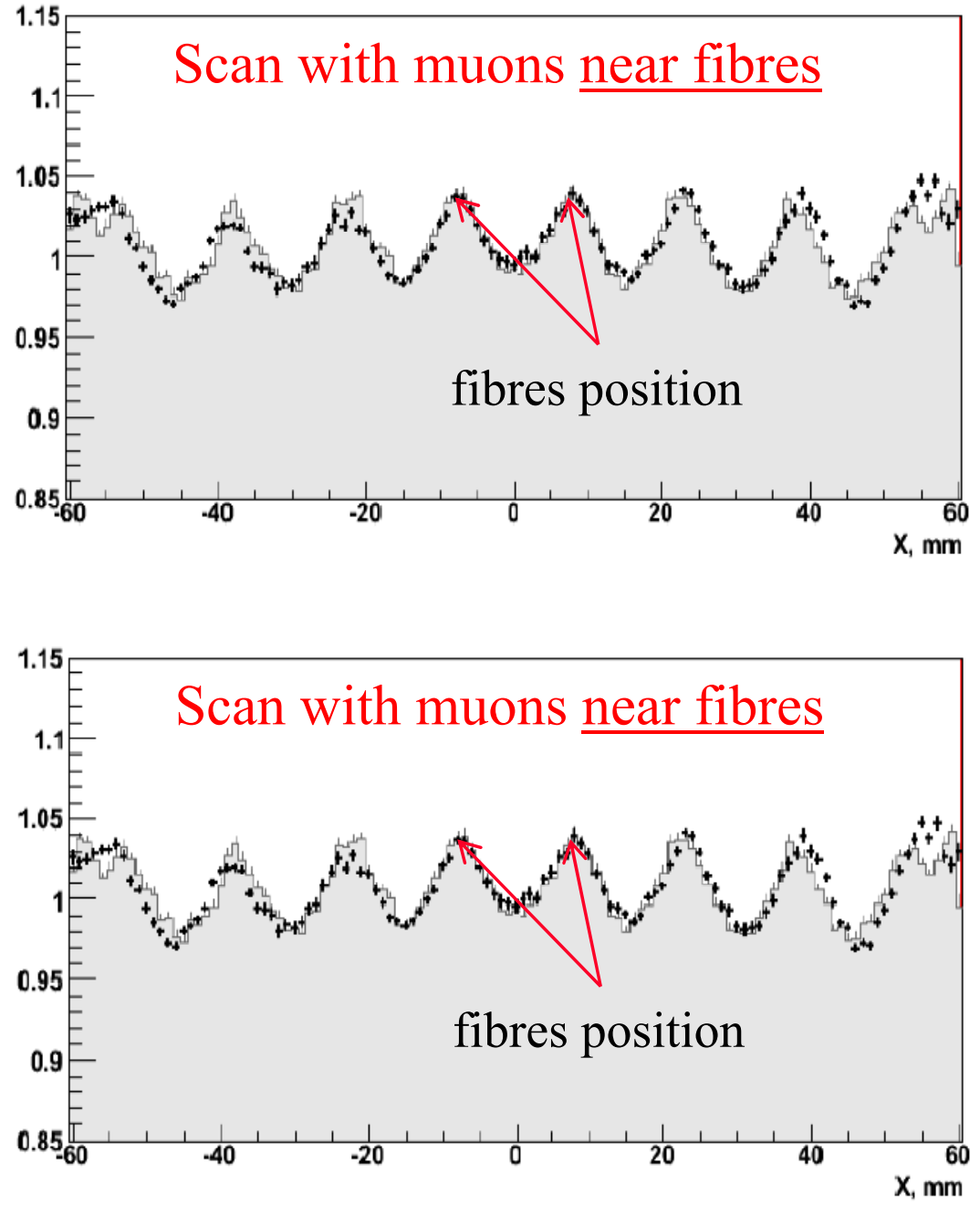


\section{ECAL structure \& integration}
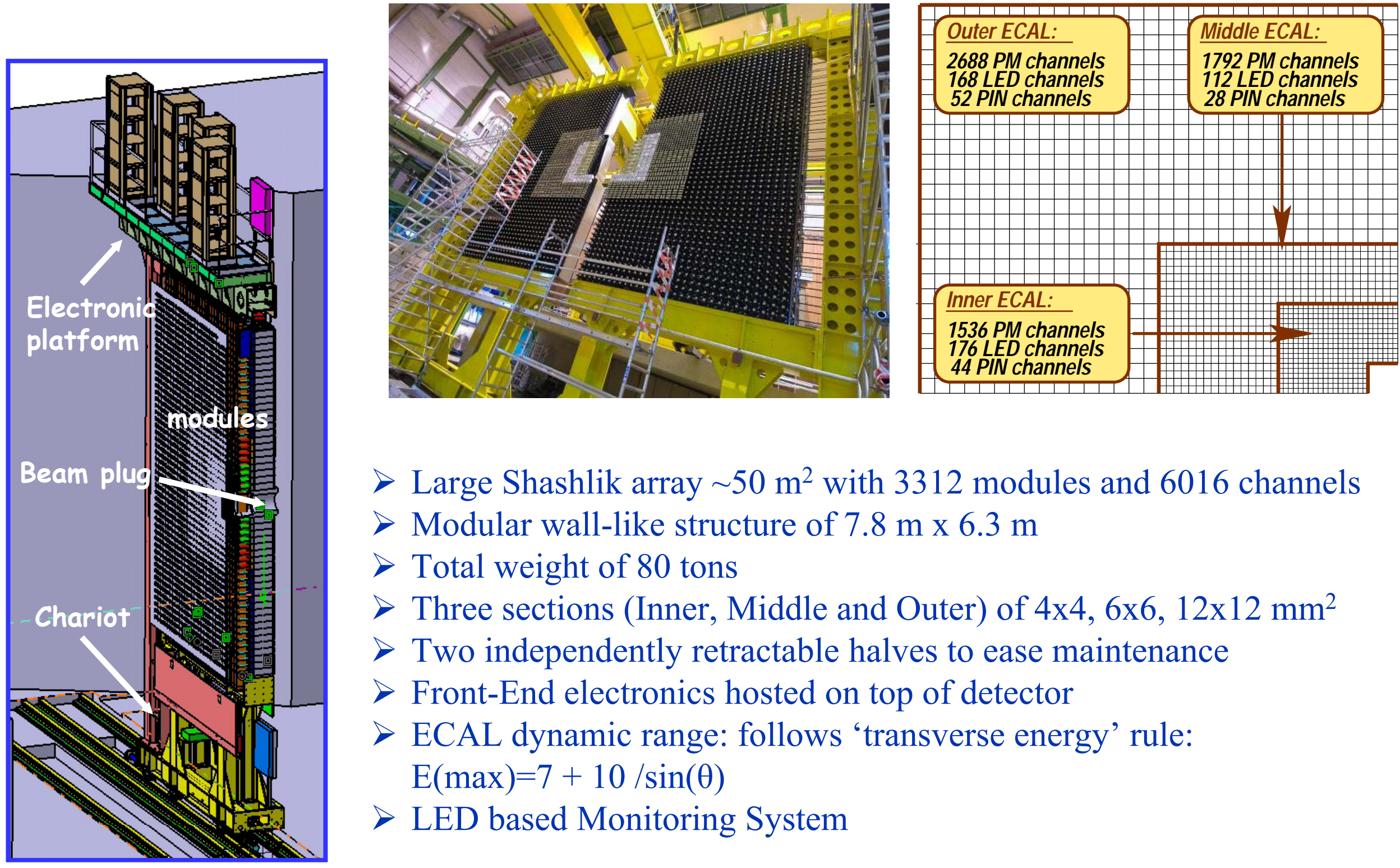

Large Shashlik array $\sim 50 \mathrm{~m}^{2}$ with 3312 modules and 6016 channels

Modular wall-like structure of $7.8 \mathrm{~m}$ x $6.3 \mathrm{~m}$

Total weight of 80 tons

$>$ Three sections (Inner, Middle and Outer) of 4x4, 6x6, 12x12 $\mathrm{mm}^{2}$

$>$ Two independently retractable halves to ease maintenance

$>$ Front-End electronics hosted on top of detector

$>$ ECAL dynamic range: follows 'transverse energy' rule: $\mathrm{E}(\max )=7+10 / \sin (\theta)$

$>$ LED based Monitoring System

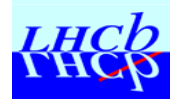




\section{LED monitoring system of ECAL}

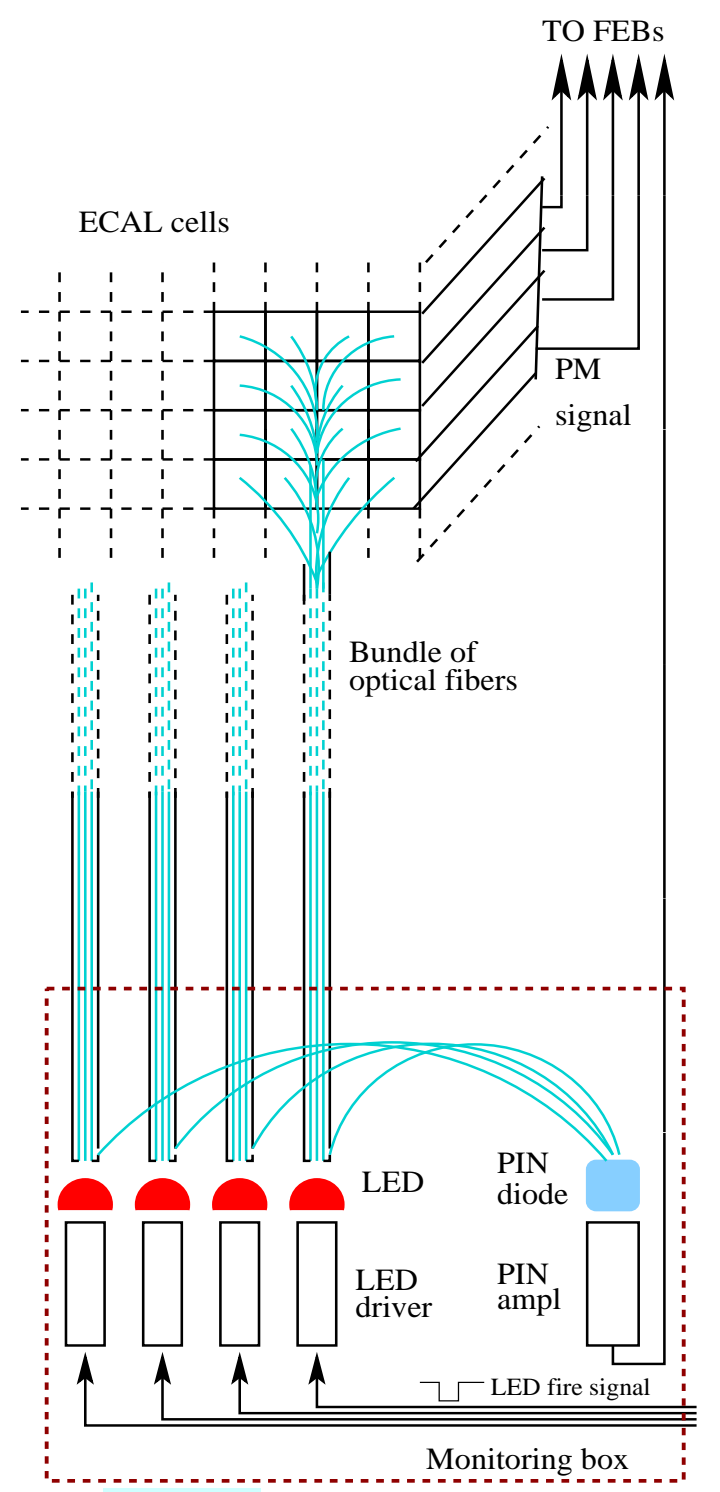

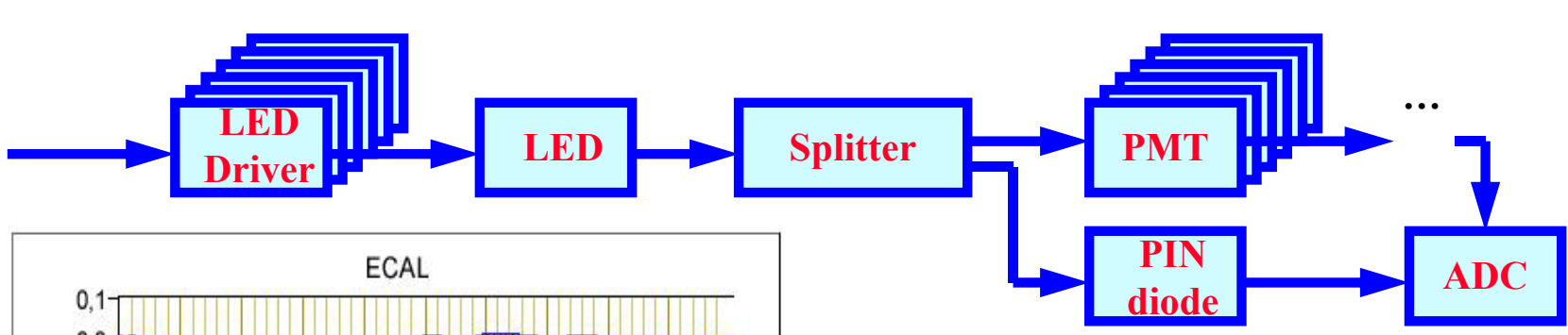

In total

> 512 LED drivers \& LEDs \& splitters \& fiber-bundles

> 64 PIN-diodes

\section{Basic requirements}

$>$ Control of time and temperature stability

$>$ Small pulse duration and dispersion of amplitude

$>$ Adjustable pulse rate and amount of light

$>$ Emulate e/m particles in full "physics" region

$>$ Gain control to better than $1 \%$ accuracy

$>$ Control only electronics chain $\rightarrow$ supply LED light directly to the PMT

$>$ Use empty bunches for running monitoring system

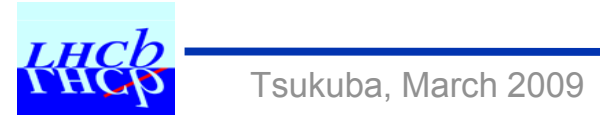


Calibration strategy of ECot

\section{$\underline{\text { Method }}$}

1. Cosmics test

2. Energy flow

3. pi0 \& e reconstruction

4. Monitoring with LED

to follow relative gain variation

Absolute calibration with resolved $\pi^{0}$

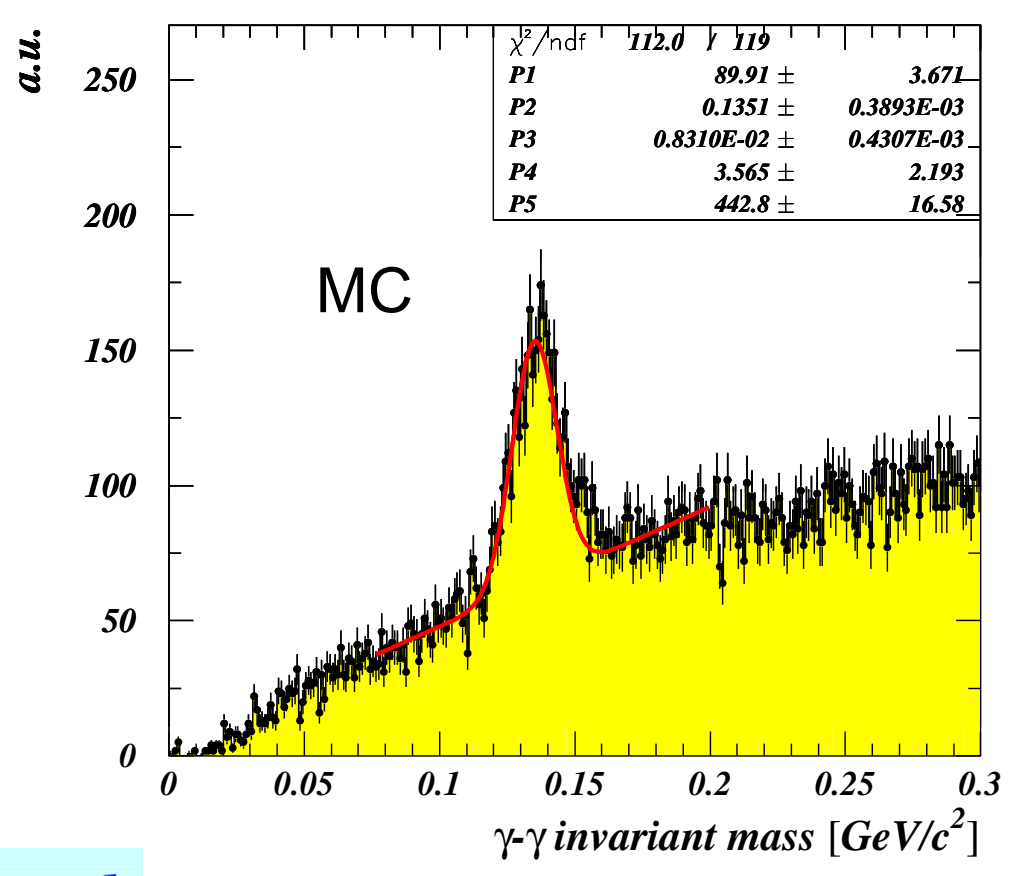

Outer section

\section{periodicitis 65 , 15}

$\sim 10 \%$

$\sim 5 \%$

$\sim 1 \%$

stability
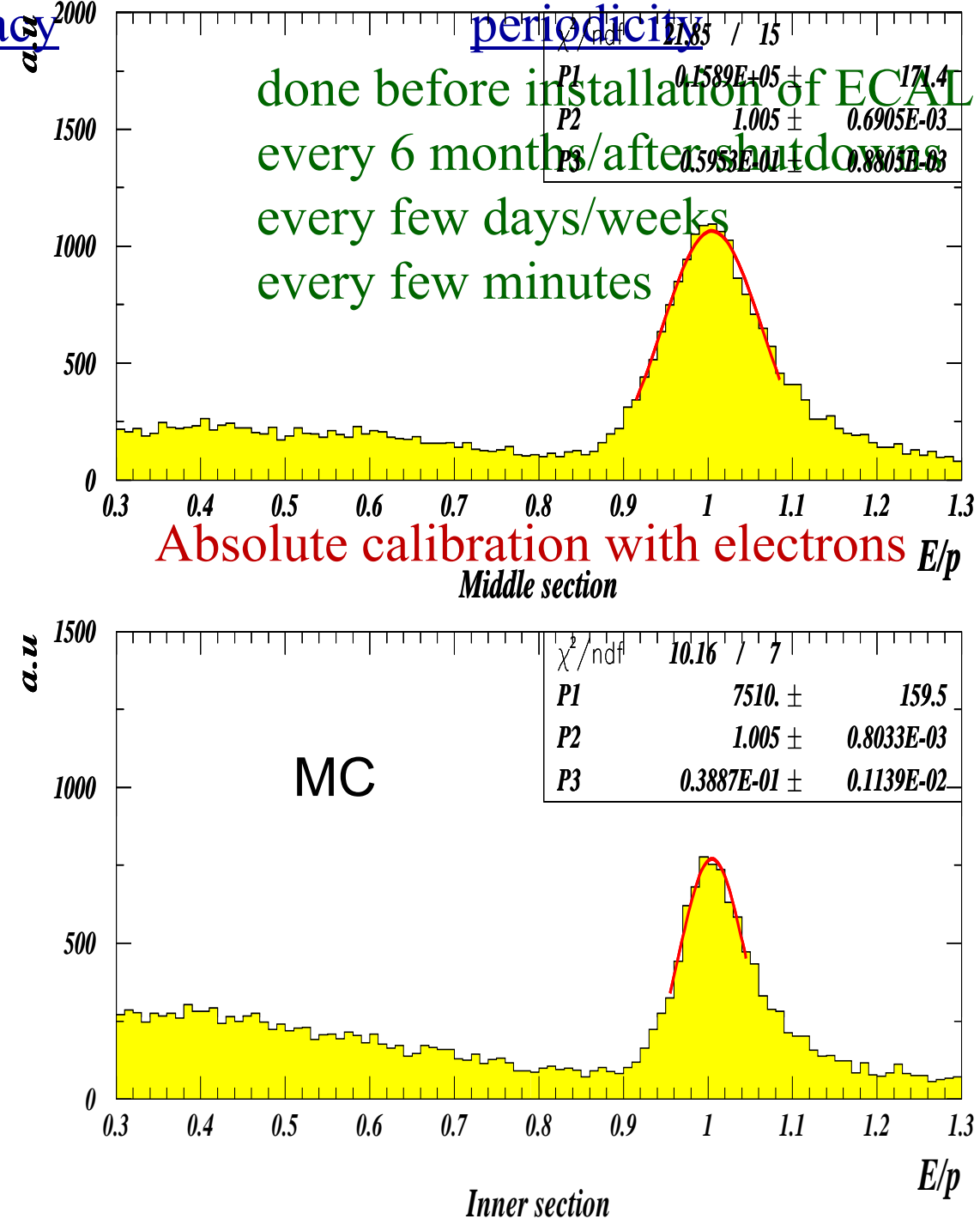


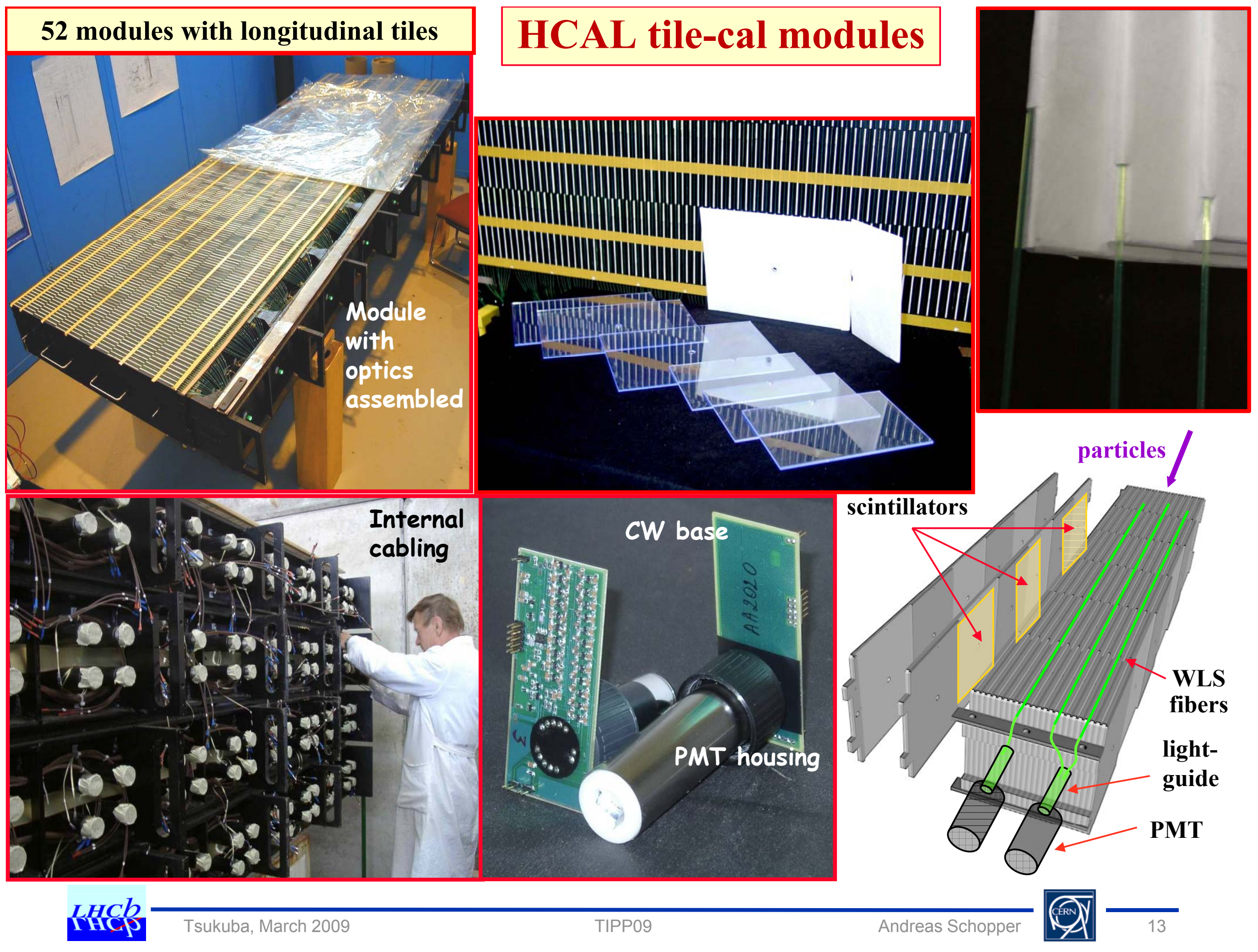




\section{HCAL module performance}

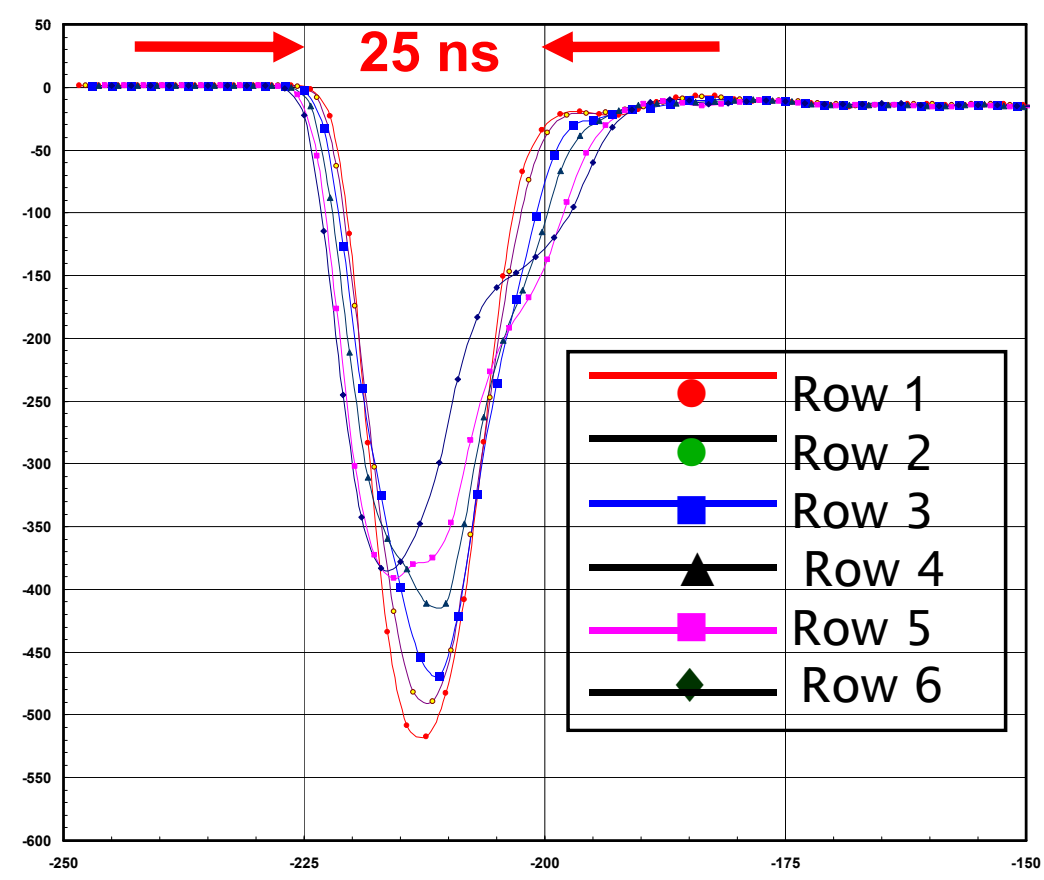

Pulse shapes from each tile row measured by scanning the HCAL module transversely with an $e^{-}$beam ( $\rightarrow$ pulse shape as function of depth)

Signal variations due to detector depth and mirrors at fiber ends
HV settings for physics: correspond to

$E_{\max }=15 \mathrm{GeV} / \sin (\Theta)$ (trigger on $E_{T}$ )

PM gains: 20k ... 350k

PM transit time $(\sim 1 / \sqrt{ } \mathrm{HV})+$ time of flight vary by $\sim 5 \mathrm{~ns}$

Signal cable delay spread: $\sim 2 \mathrm{~ns}$

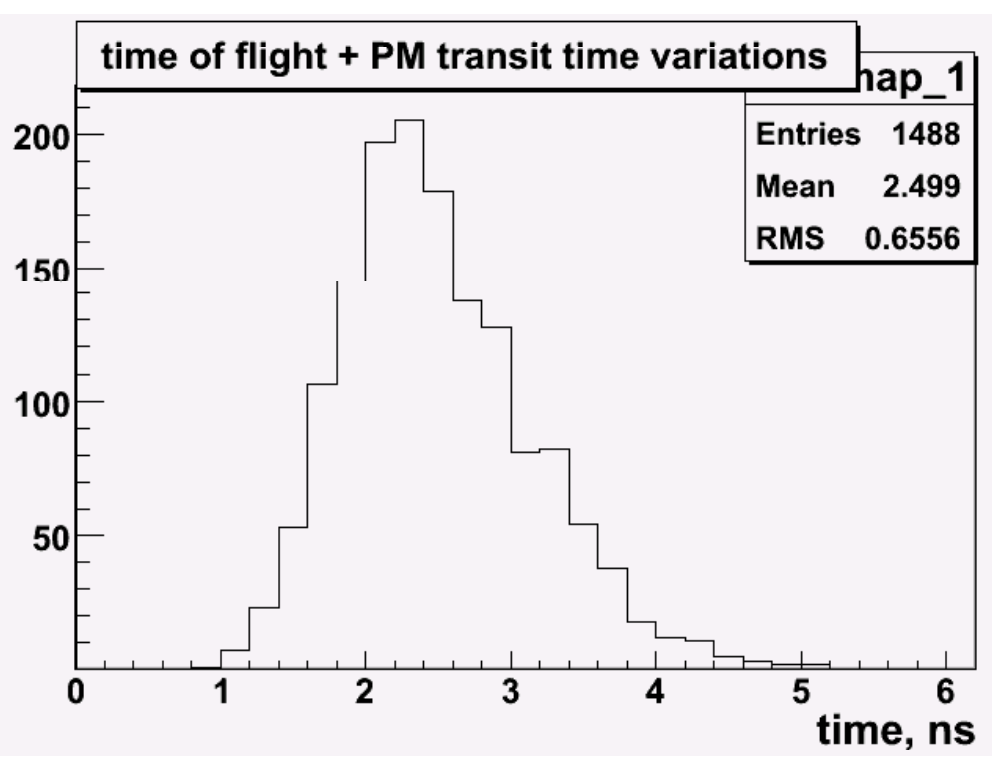




\section{HCAL module performance}

\section{Energy resolution}

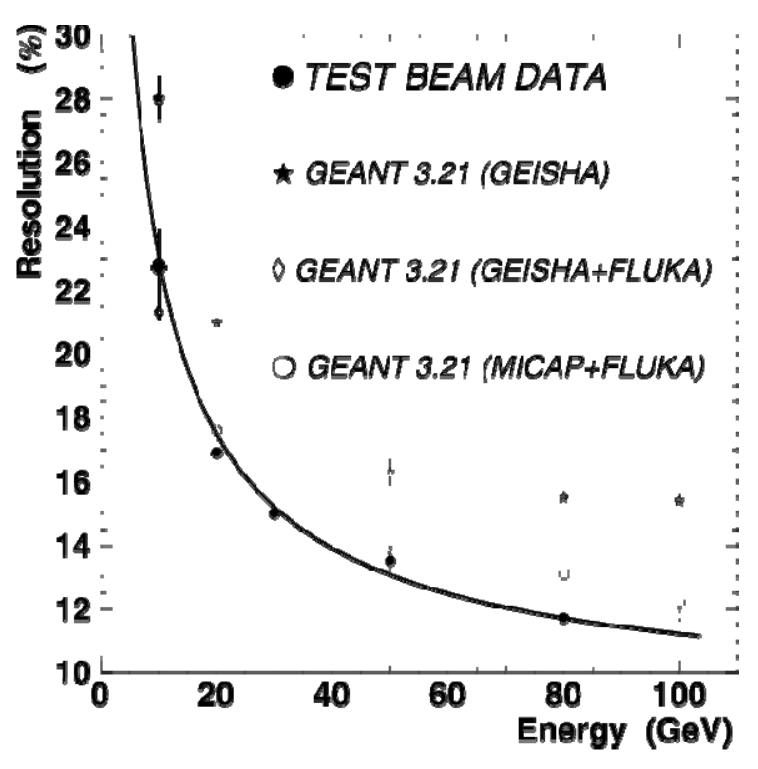

$\frac{\sigma}{E}=\frac{(69 \pm 5) \%}{\sqrt{E}} \oplus(9 \pm 2) \%$
Angular dependence

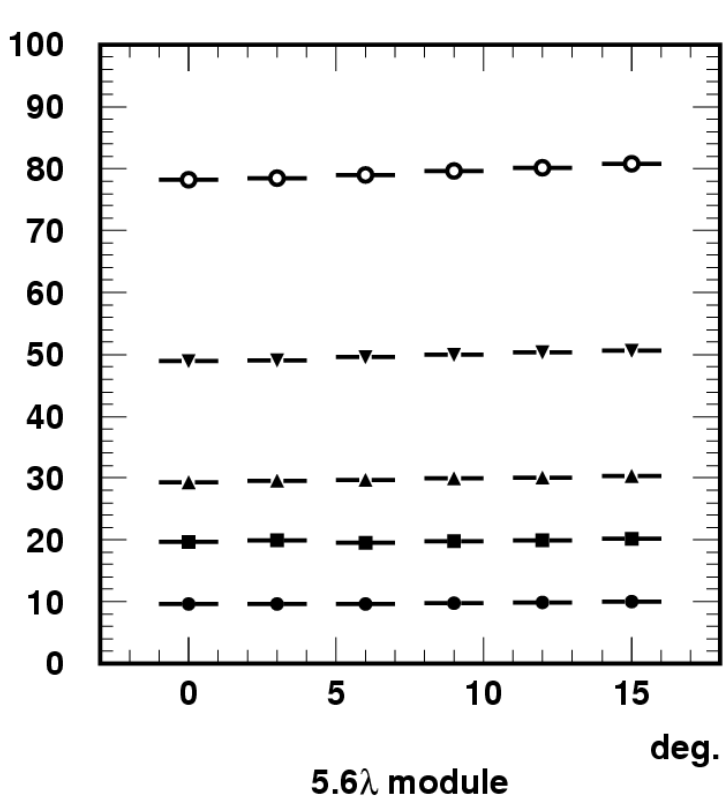

$\sim 3 \%$ angular dependence at higher energies: shower not fully contained in $5.6 \lambda_{\mathrm{I}}$

\section{Light yield}

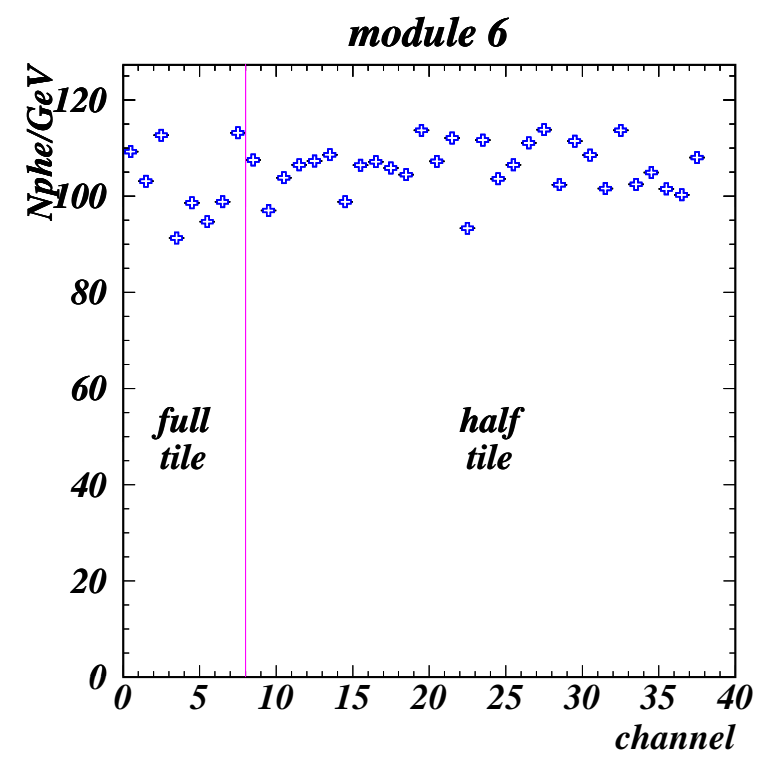

Average light yield: 105 ph.el./GeV 


\section{HCAL structure \& integration}

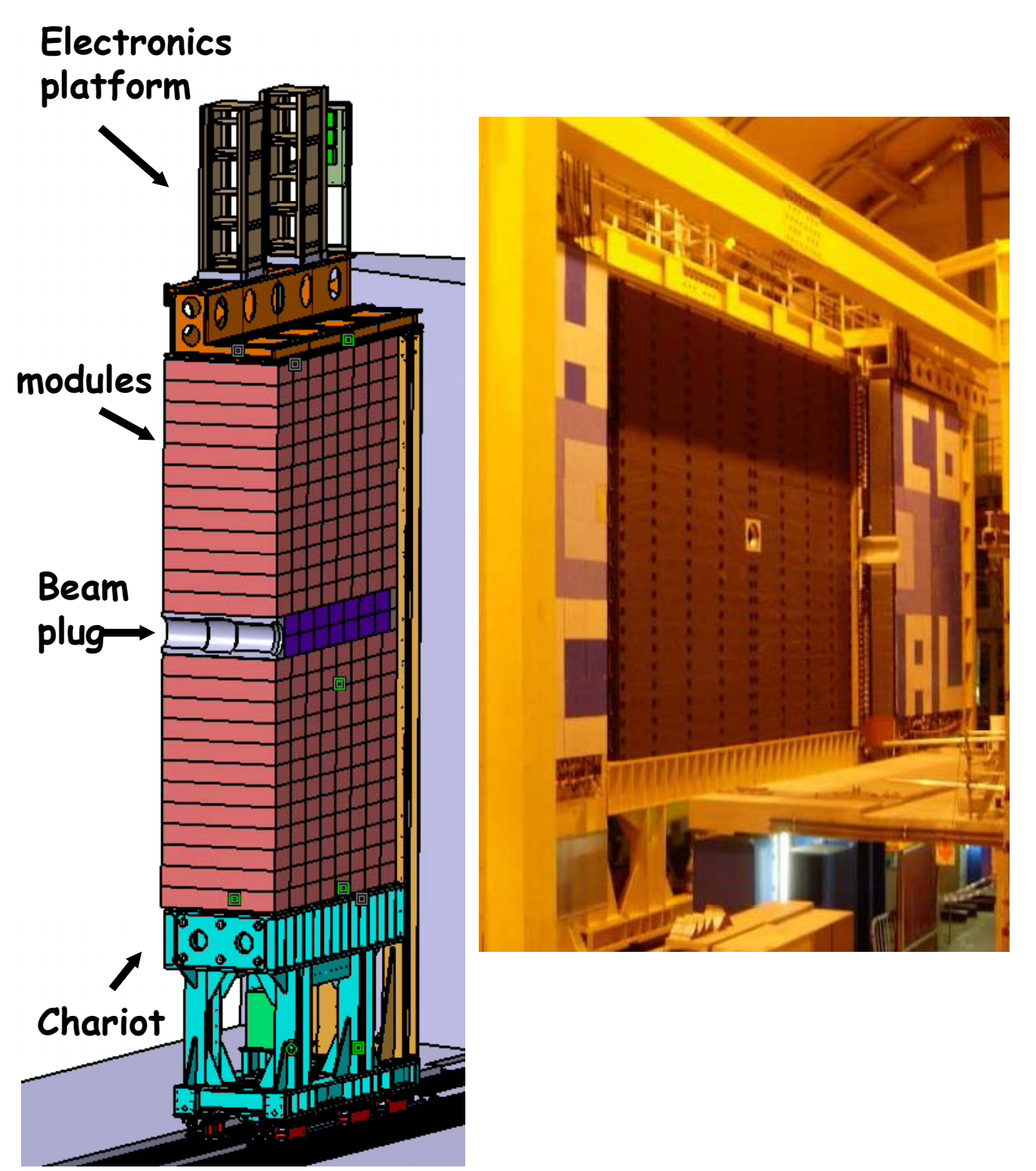

> Two independently retractable halves each consisting of 26 modules stacked on a movable platform

$>$ size of active area: $8.4 \times 6.8 \mathrm{~m}^{2}$

$>$ instrumented depth: $120 \mathrm{~cm}$

$>$ cell size:

- outer zone 262 x $262 \mathrm{~mm}^{2}$

- inner zone $131 \mathrm{x} 131 \mathrm{~mm}^{2}$

1488 cells (608 outer +880 inner)

- LED based Monitoring System

$>$ built-in ${ }^{137} \mathrm{Cs}$ calibration system for in situ calibration

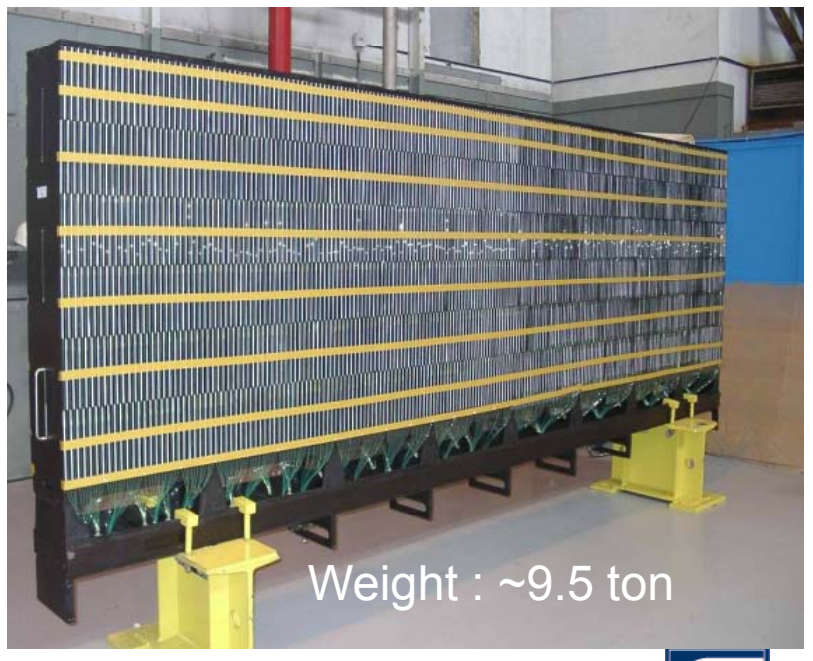

KHCh Tsukuba, March 2009

TIPP09

Andreas Schopper

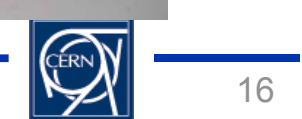




\section{LED monitoring system of HCAL}
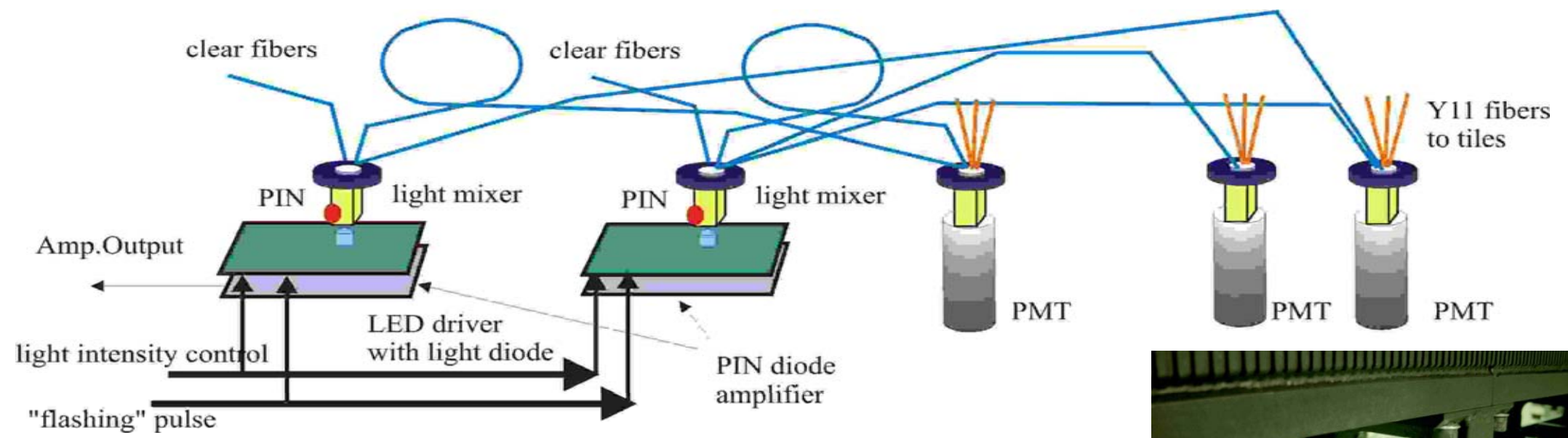

$>$ blue LEDs (WU-14-750BC)

two independent LEDs per module adjustable LED pulse amplitude

$>$ monitoring PIN photodiode at each LED in order to account for LED instability

$>$ light distribution with clear fibers of same length

timing of the LED flashing pulse adjustable with 1 ns step
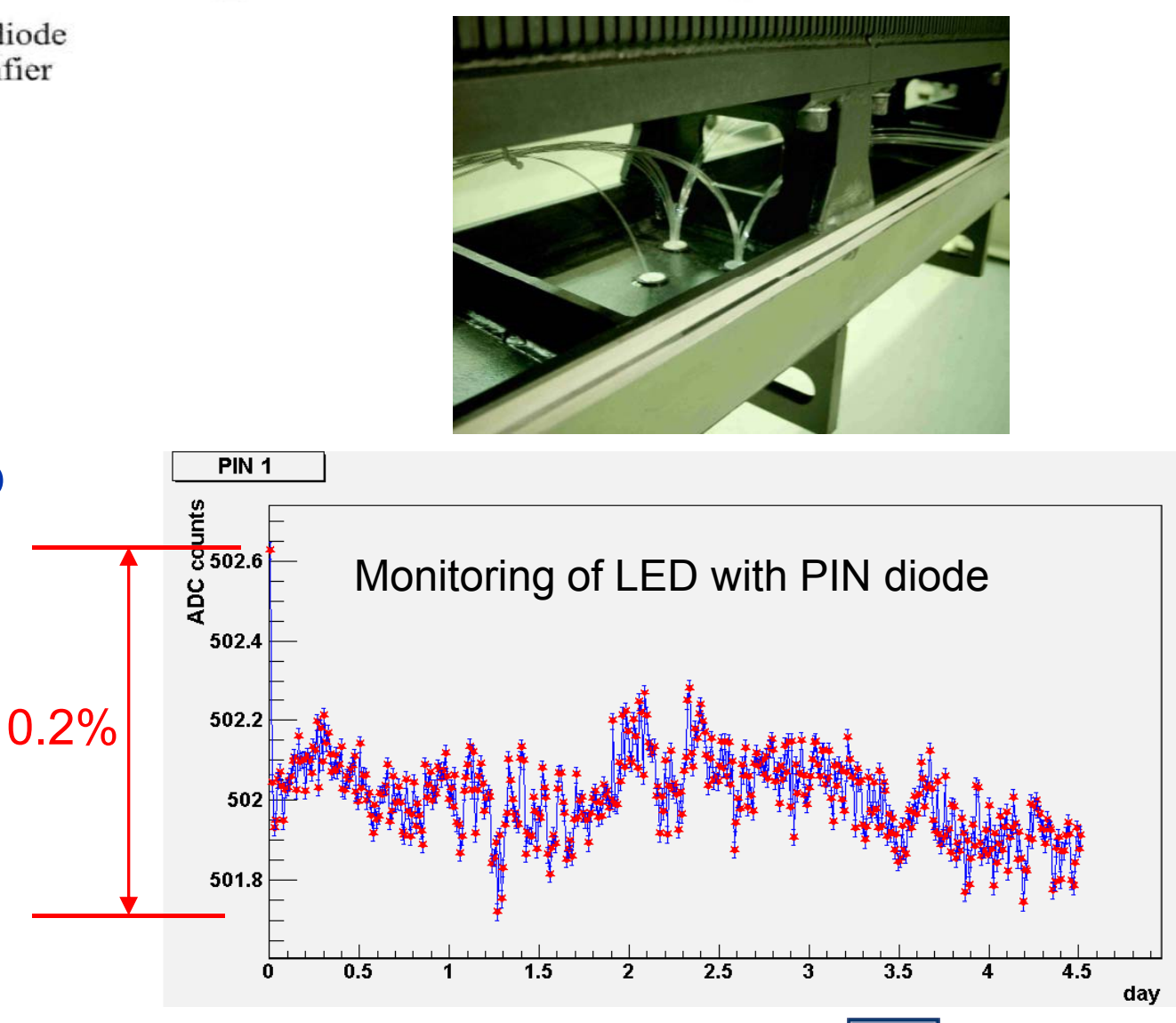


\section{Calibration system of HCAL}
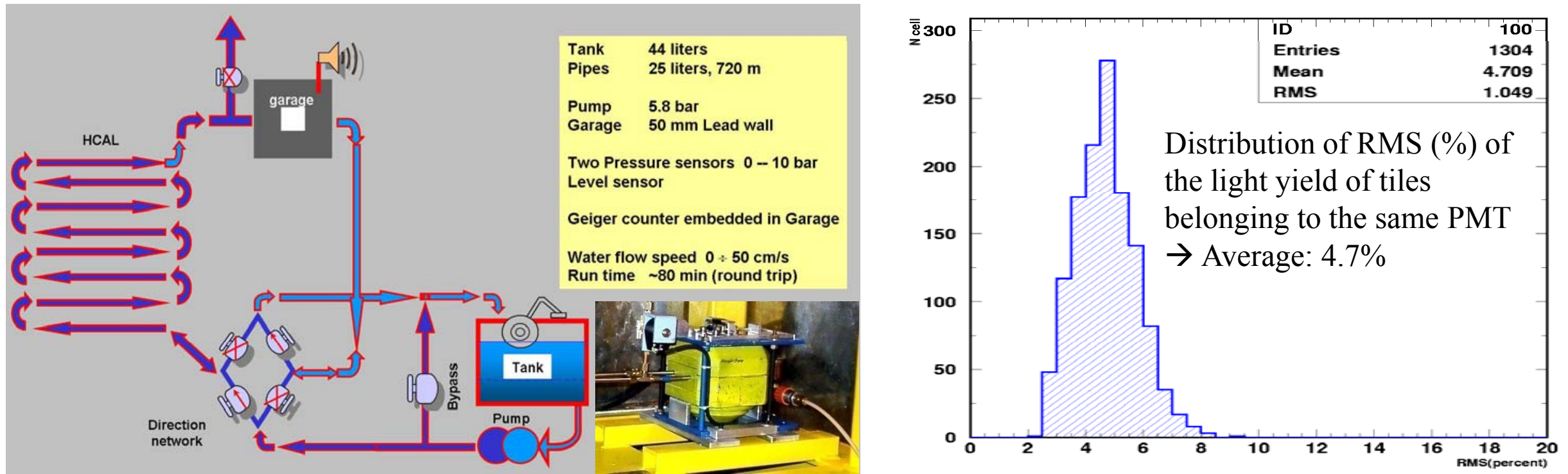

\section{[PMT 13] Mean= 581 Fibr.RMS $=4.3 \%$ Perc $=20 \%$ Left-Top'}

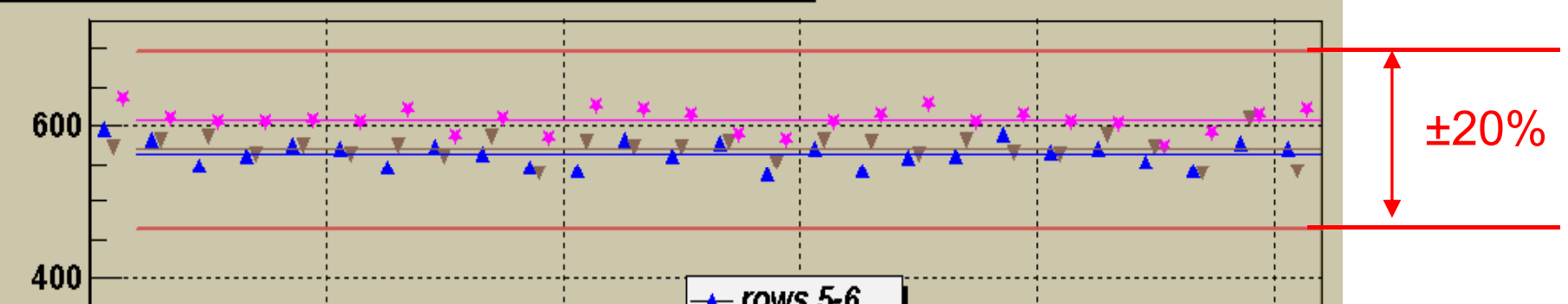

\section{$\rightarrow$ rows 3-4 \\ -7 rows 1-2}

All HCAL modules have undergone Cs test during production with requirement that tile response has to be within $\pm 20 \%$ of average

0
0

Testbeam measurements show that independent calibrations with $\mathrm{Cs}$ source and $50 \mathrm{GeV} \pi^{-}$coincide within $2-3 \%$ 


\section{ECAL \& HCAL readout electronics}
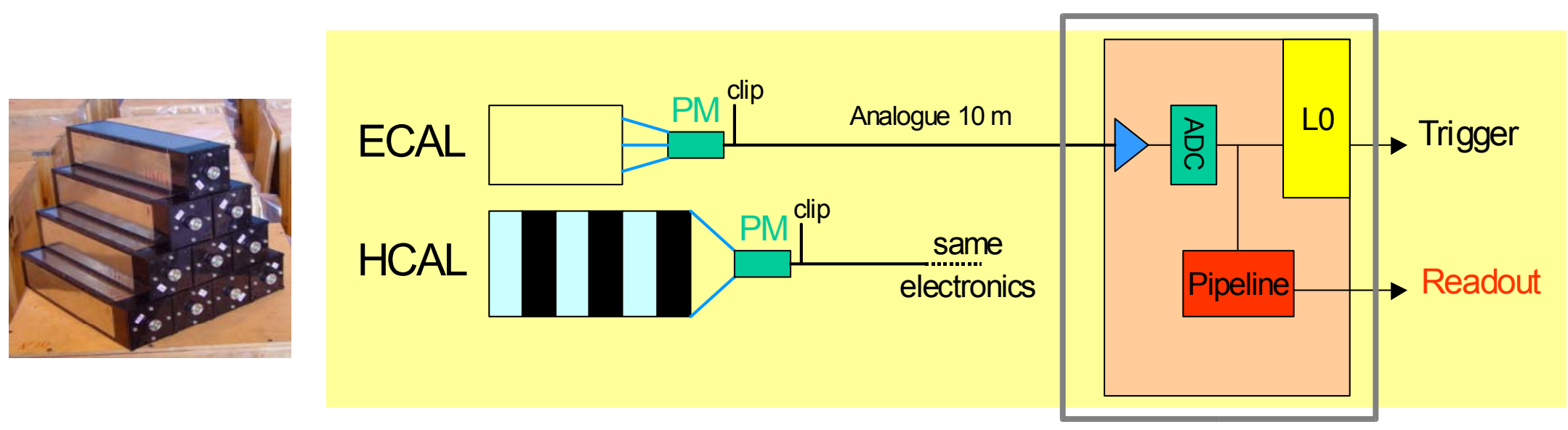

$\checkmark \quad$ Signal processing

$>$ Shaper integrator +12 -bit $(80 \mathrm{pC}) 40 \mathrm{MHz}$ two-stage bipolar flash ADC

+ delay chip to compensate for different arrival times of PM signals

$>$ pedestal subtraction (suppression of low-frequency noise)

$>$ conversion to 8-bit $\mathrm{E}_{\mathrm{t}}$ calibrated signal

$\checkmark$ Selection of cluster candidate with highest $\mathrm{E}_{\mathrm{t}}$ for L0

$\checkmark$ Formatting of data block and dispatch it to the DAQ (upon request) 


\section{SPD \& PS choice of technology}

Purpose of SPD \& PS:

$>$ PS : electron/pion separation

$>$ SPD : photon/mip separation

$>$ part of very first trigger level at $40 \mathrm{MHz}$

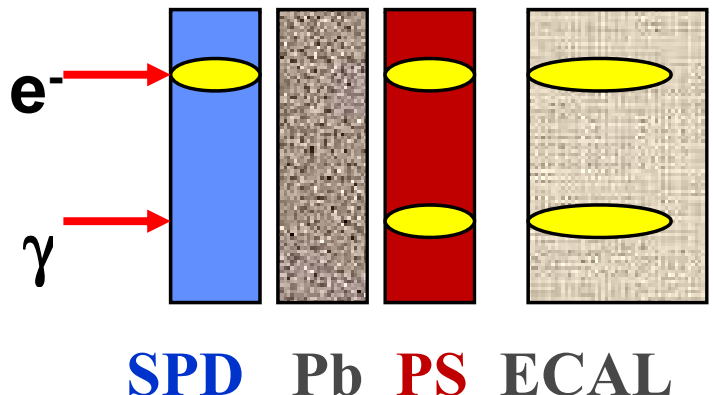

SPD \& PS design requirements:

fast response time compatible with LHC bunch spacing of $25 \mathrm{~ns}$

$>$ match transverse segmentation of ECAL

$>$ full projectivity and minimal distance between SPD and PS for efficient trigger

$>$ lateral size of active area $7.8 \mathrm{~m} \times 6.3 \mathrm{~m}$

$>$ radiation hard up to $\sim 150 \mathrm{krad} /$ year

$>$ cost effective

$>$ space constraint of $18 \mathrm{~cm}$ in depth (along beam pipe)!

$\rightarrow$ Two layers of scintillator interspaced by $2.5 \times 0$ lead with a total of 12 '032 channels

$\rightarrow$ Light transported via clear fibers to detector periphery, where PMTs are located 

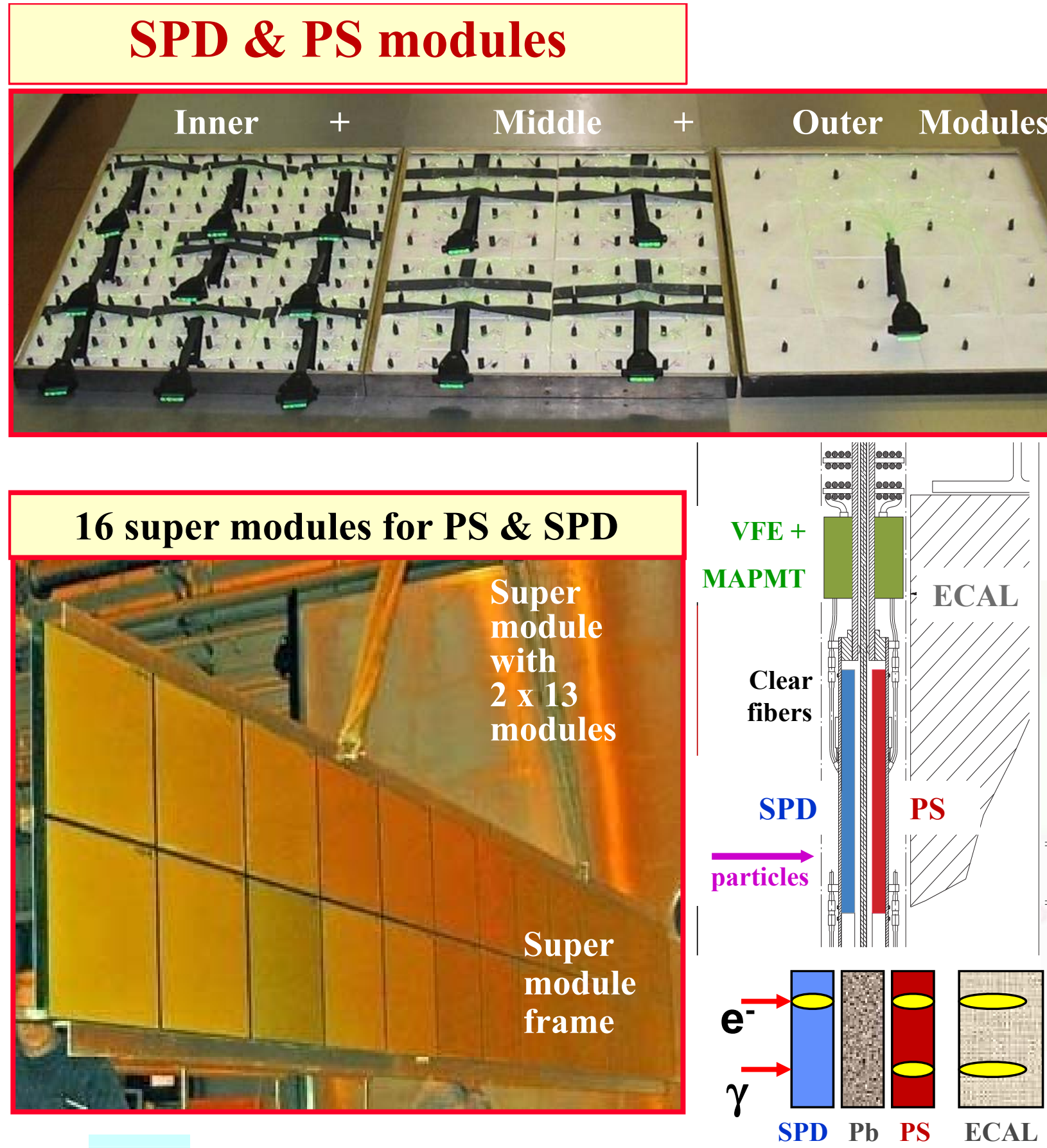

\section{Scintillator + coiled fiber}

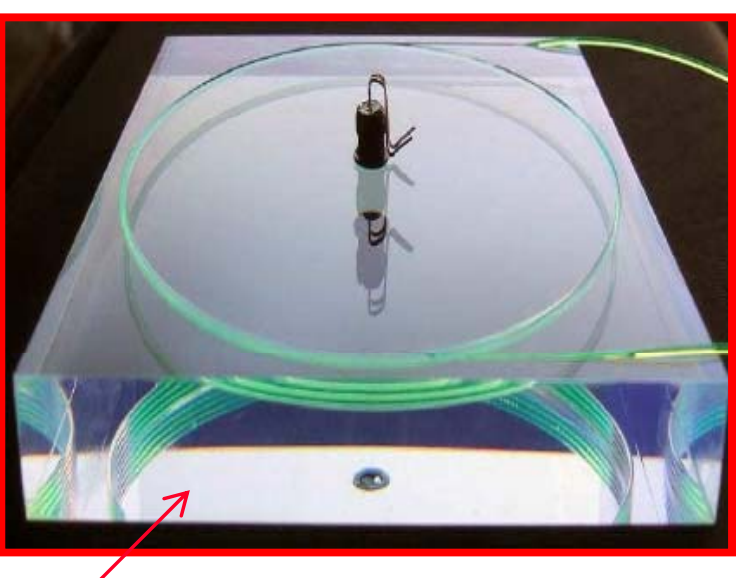

thickness $15 \mathrm{~mm}$

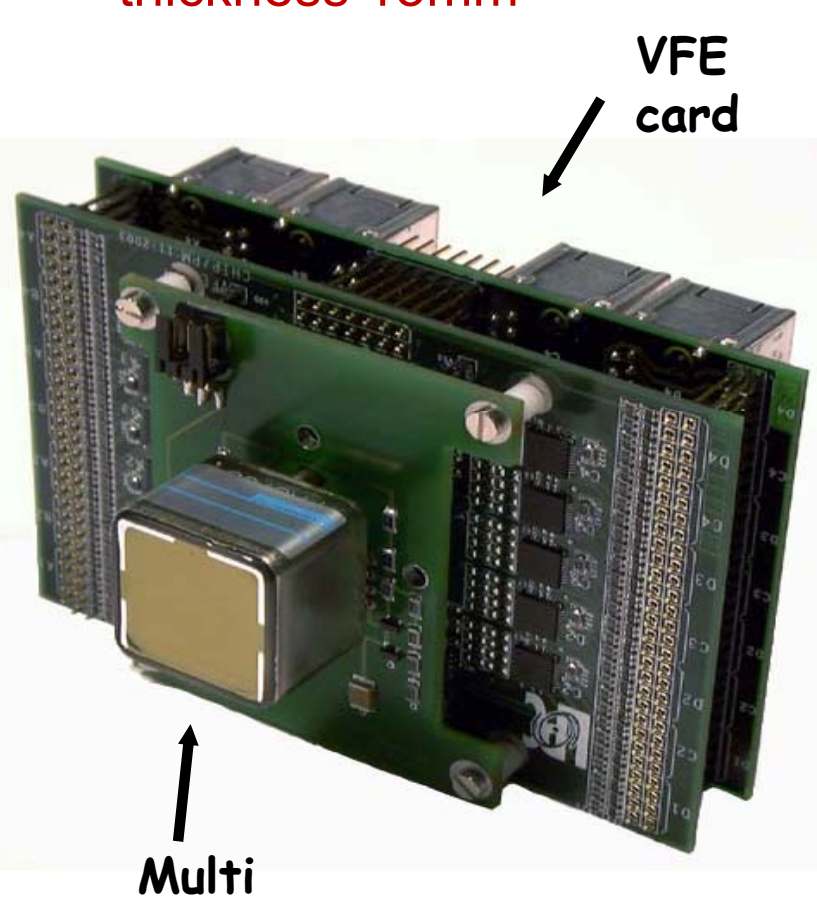

Anode

PMT

Andreas Schopper

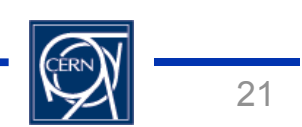




\section{SPD \& PS module performance}

Optimize light yield from

$>15 \mathrm{~mm}$ thick tile with coiled WLS fiber $+\sim 3 \mathrm{~m}$ long clear fibers and interconnects

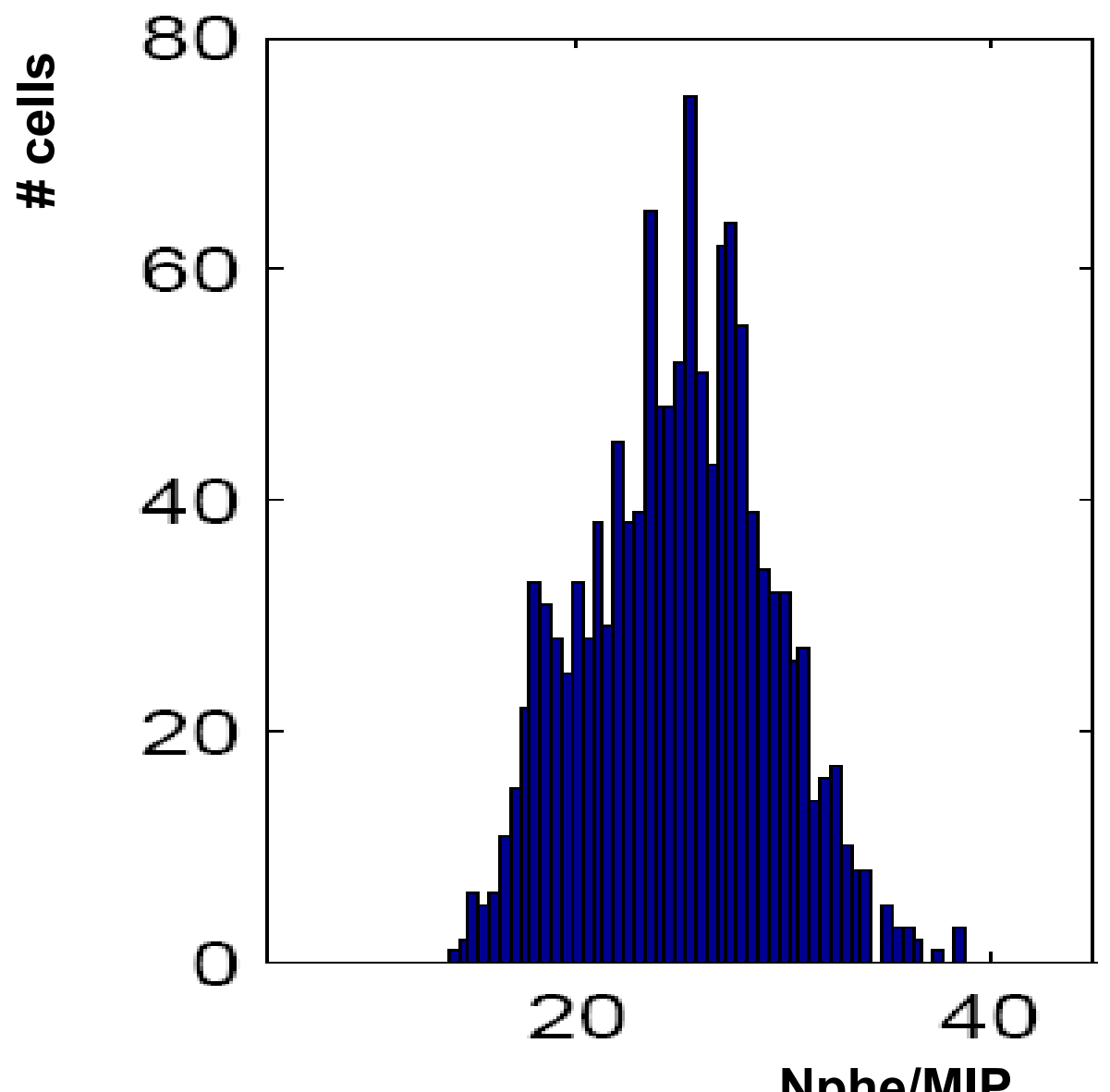

All super-modules tested with

cosmics in horizontal position

$\rightarrow \sim 25 \pm 15$ photo-electrons per MIP

PS:

$>$ Readout by 10 bit ADC

$>$ Noise below 1.2 ADC counts with $\sim 10$ ADC-counts/MIP

SPD:

$>$ Digital readout (1 bit)

$>$ Noise below $3 \mathrm{mV}$ with 100mV/MIP

Nphe/MIP 


\section{Calibration of SPD \& PS}
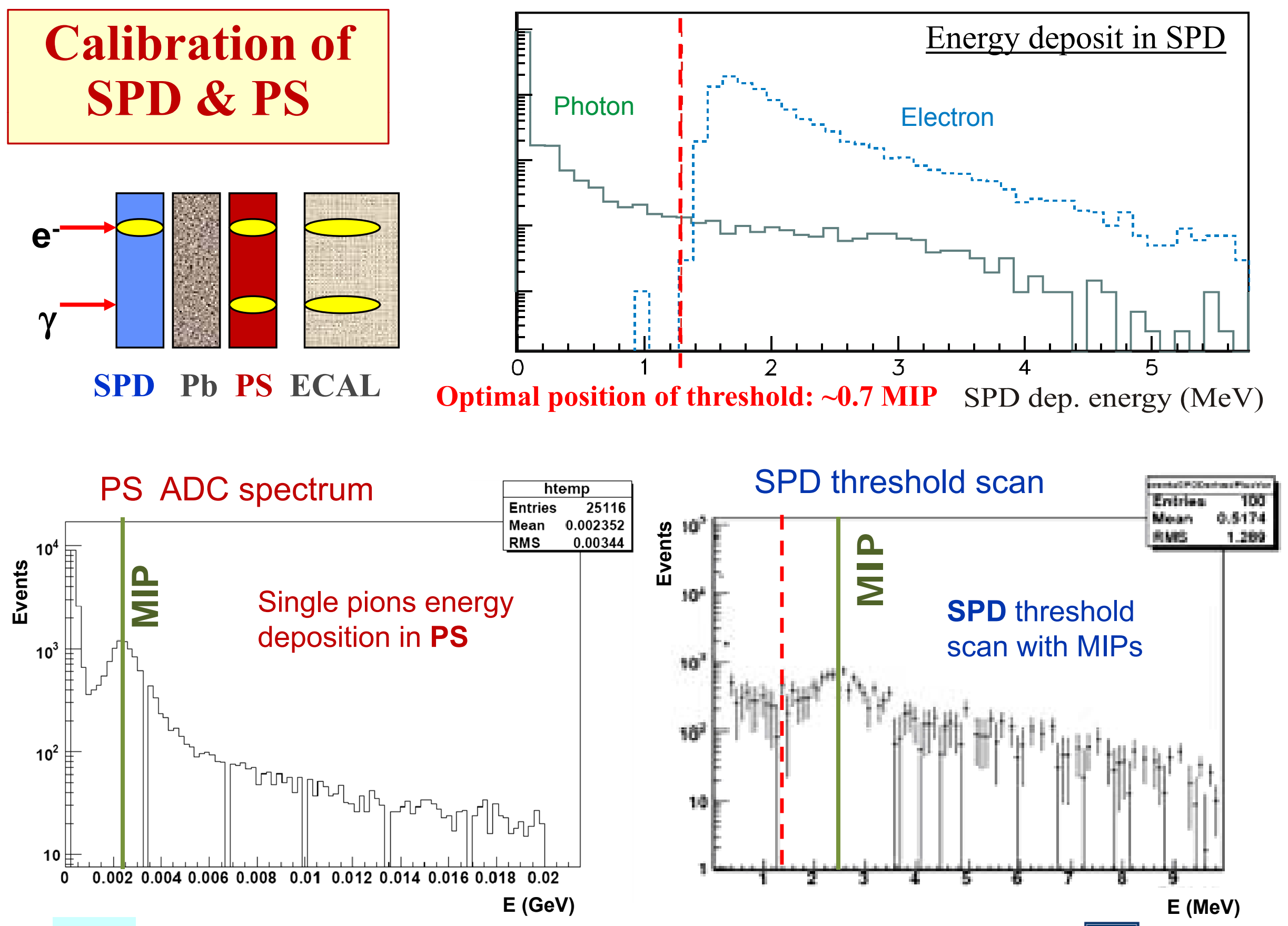

LHCP Tsukuba, March 2009 


\section{SPD \& PS structure \& integration}

$>$ PS+SPD built from a total of 16 super modules

$>$ segmentation matches (projective) ECAL cell size

$>$ total of $12^{\prime} 032$ channels
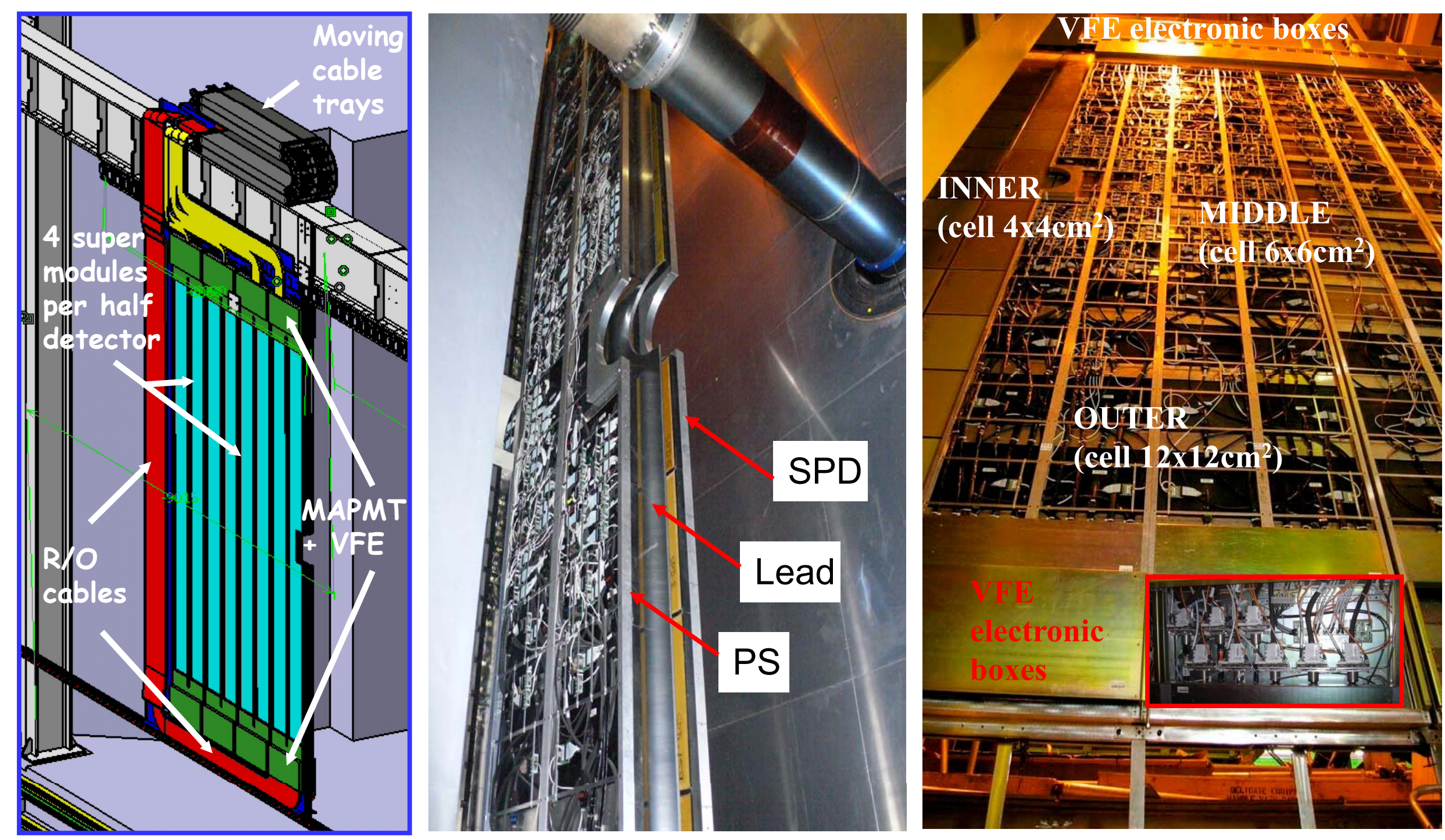


\section{Monitoring of SPD \& PS with LED and particles}

Each of the $12^{\prime} 032$ cells is equipped with a LED

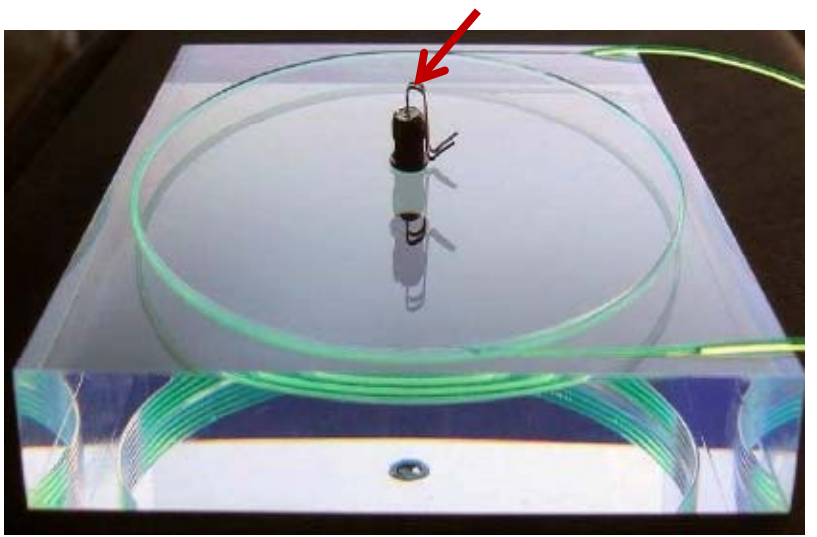

LED signals used:

$>$ to detect dead channels

$>$ to check the detector stability

$>$ for coarse time alignment
2D display during commissioning of PS showing peak ADC value of LED flash with subtracted pedestal

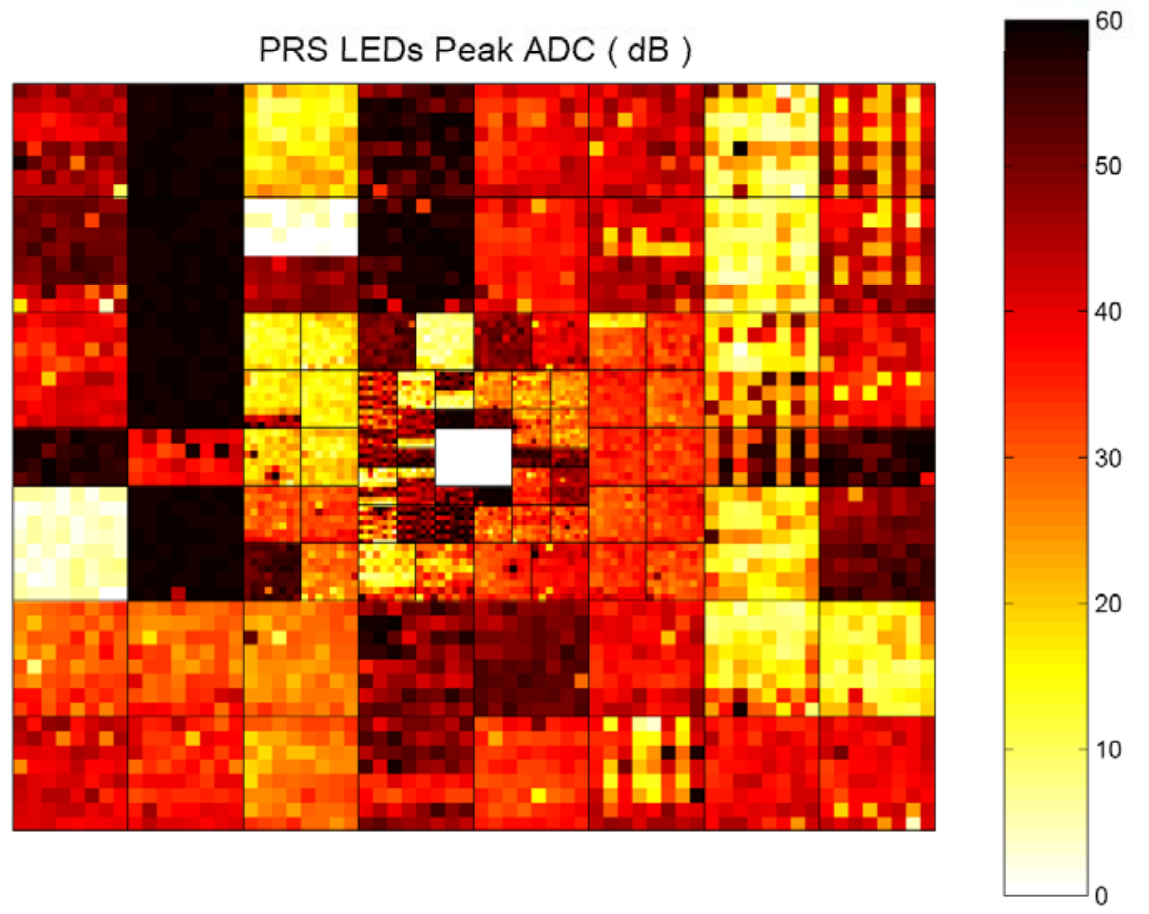

$5 \mathrm{~Hz}$ online event reconstruction to monitor with particles

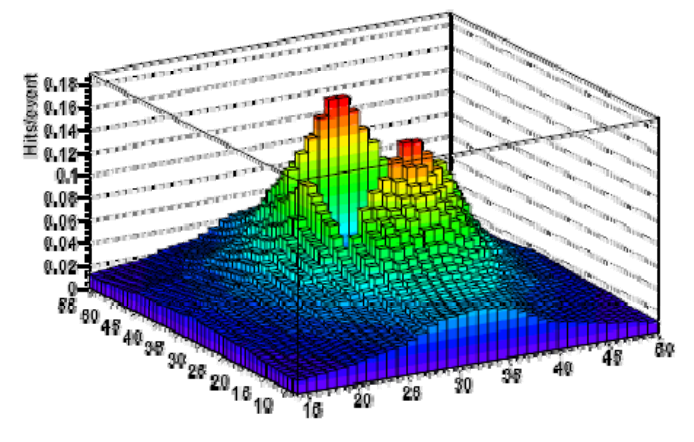

Normalize to neighboring cells

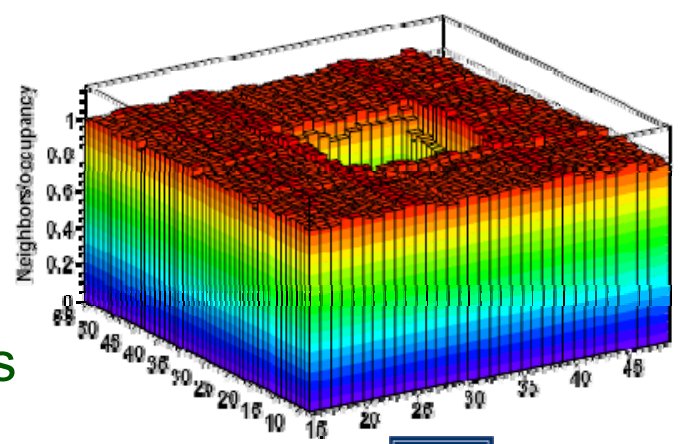




\section{PS \& SPD readout electronics}
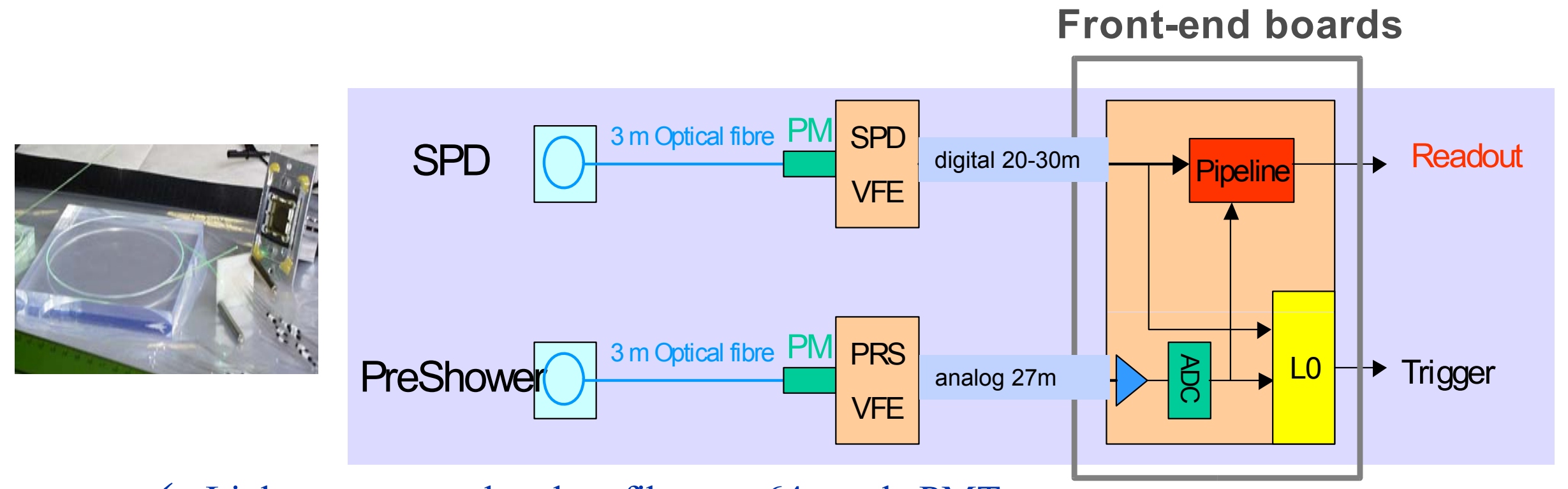

$\checkmark$ Light transporter by clear fibers to 64 anode PMT

$>$ very front-end away from beam : no radiation problem

$\checkmark$ Dynamic range : $0-100$ MIPs and accuracy required $\sim 10 \%$

$>$ PS : electron/pion separation $\rightarrow 10$ bits

$>\mathrm{SPD}:$ photon/mip separation $\rightarrow 1$ bit

$\checkmark \quad 25 \pm 15$ photo-electrons per MIP $\rightarrow$ large fluctuations

$\checkmark 25$ ns integrator mounted on the PMT - Reset with switches

$>$ Cheap and maximum use of the photo-electrons

$>$ Potentially more sensitive to noise, drift of pedestal and switch time versus beam crossing 


\section{Illustration : physics with prompt photons}

e.g. radiative $\mathrm{B}$ decays

$$
\sigma\left(\mathrm{M}_{\mathrm{B}}\right) \sim 65 \mathrm{MeV} / \mathrm{c}^{2}
$$

\begin{tabular}{|c|}
\hline $\mathrm{B}_{\mathrm{d}} \rightarrow \mathrm{K}^{*} \mathrm{~V} \quad\left(\mathrm{Br}=2.9 \times 10^{-5}\right)$ \\
$35 \times 10^{3}$ selected evts/year $-\mathrm{B} / \mathrm{S}<0.7$ \\
\hline $\mathrm{B}_{\mathrm{S}} \rightarrow \phi \gamma \quad\left(\mathrm{Br}=4.3 \times 10^{-5}\right)$ \\
$9 \times 10^{3}$ selected evts/year $-\mathrm{B} / \mathrm{S}<2.4$ \\
\hline
\end{tabular}

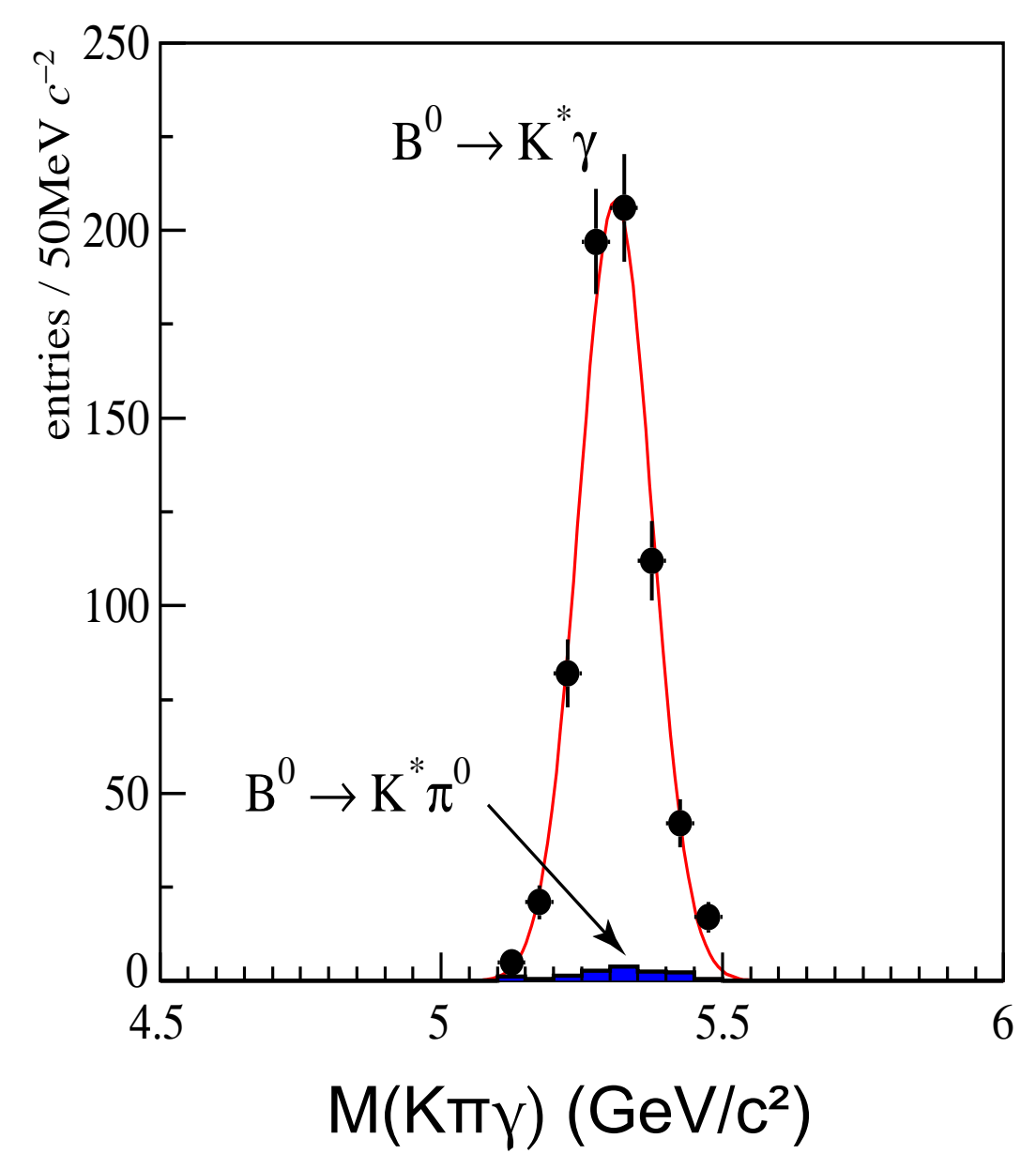




\section{Illustration : physics with neutral pions}

Efficiency to reconstruct $\pi^{0}$ in $\mathrm{B}_{\mathrm{d}} \rightarrow \pi^{+} \pi^{-} \pi^{0}$ events

$$
\varepsilon=53 \%
$$

(33\% from resolved $+20 \%$ from merged)

Most of the inefficiency due to photon conversion before magnet

$$
\mathrm{B}_{\mathrm{d}} \rightarrow \pi^{+} \pi^{-} \pi^{0} \quad\left(\mathrm{Br}=2.010^{-5}\right)
$$

12. $10^{3}$ selected evts/year $-\mathrm{B} / \mathrm{S}<1$

$\sim 60 \%$ with merged $\pi^{0} \rightarrow \mathrm{VY}$

$B_{u} \rightarrow \rho^{+} \rho^{0}$

$$
(\mathrm{Br}=2.0 \text { 10-5) }
$$

19. $10^{3}$ selected evts/year $-\mathrm{B} / \mathrm{S}<1$

$\sim 30 \%$ with merged $\pi^{0} \rightarrow \gamma \gamma$

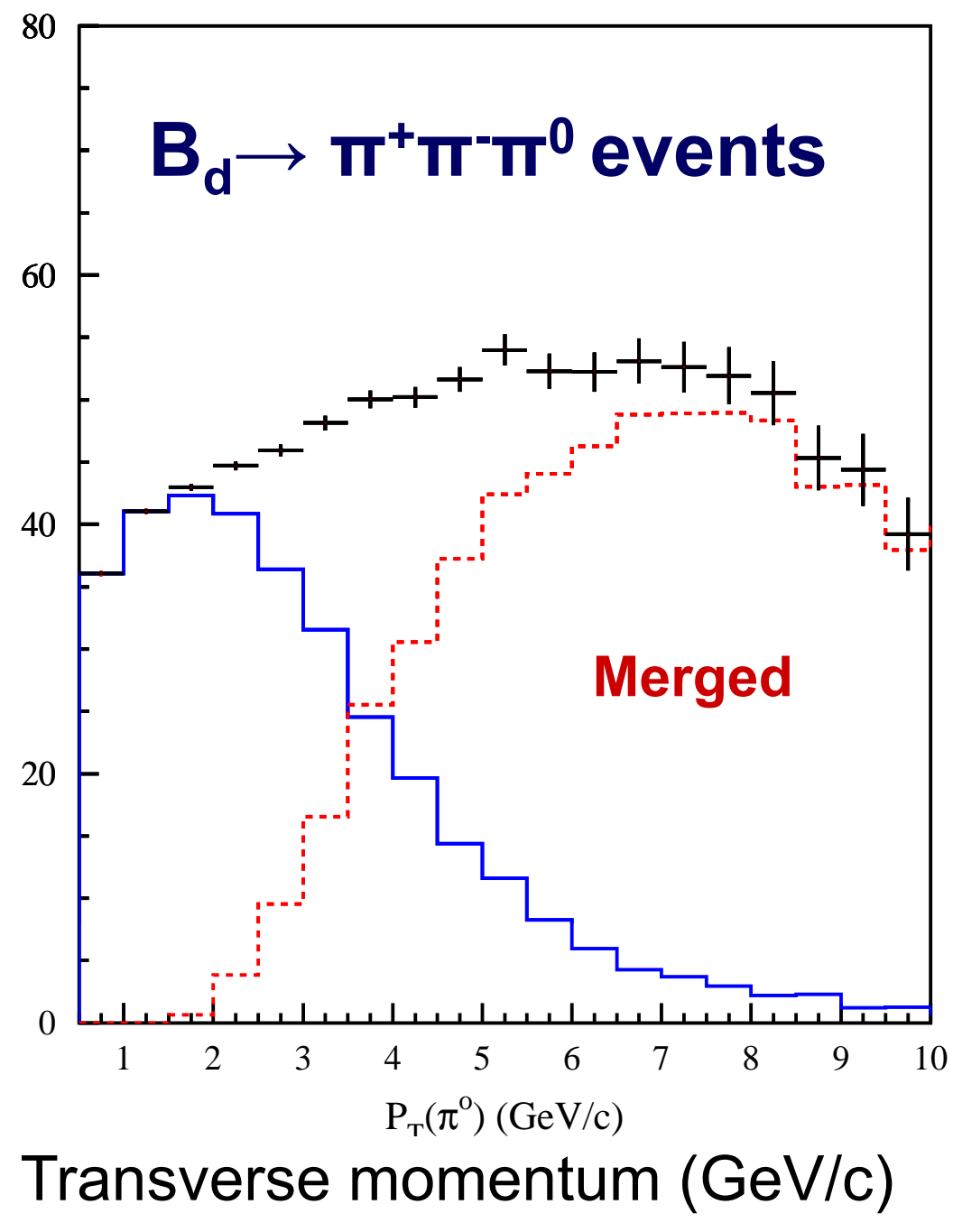




\section{Commissioning with Cosmics}

LHCb geometry NOT well suited for cosmics... A challenge!

Calorimeters used to trigger on useful 'horizontal' cosmics

$\rightarrow$ Rate of 'horizontal' cosmics below $1 \mathrm{~Hz}$, still very useful

Collected a total of $\sim 1.1$ Million triggers

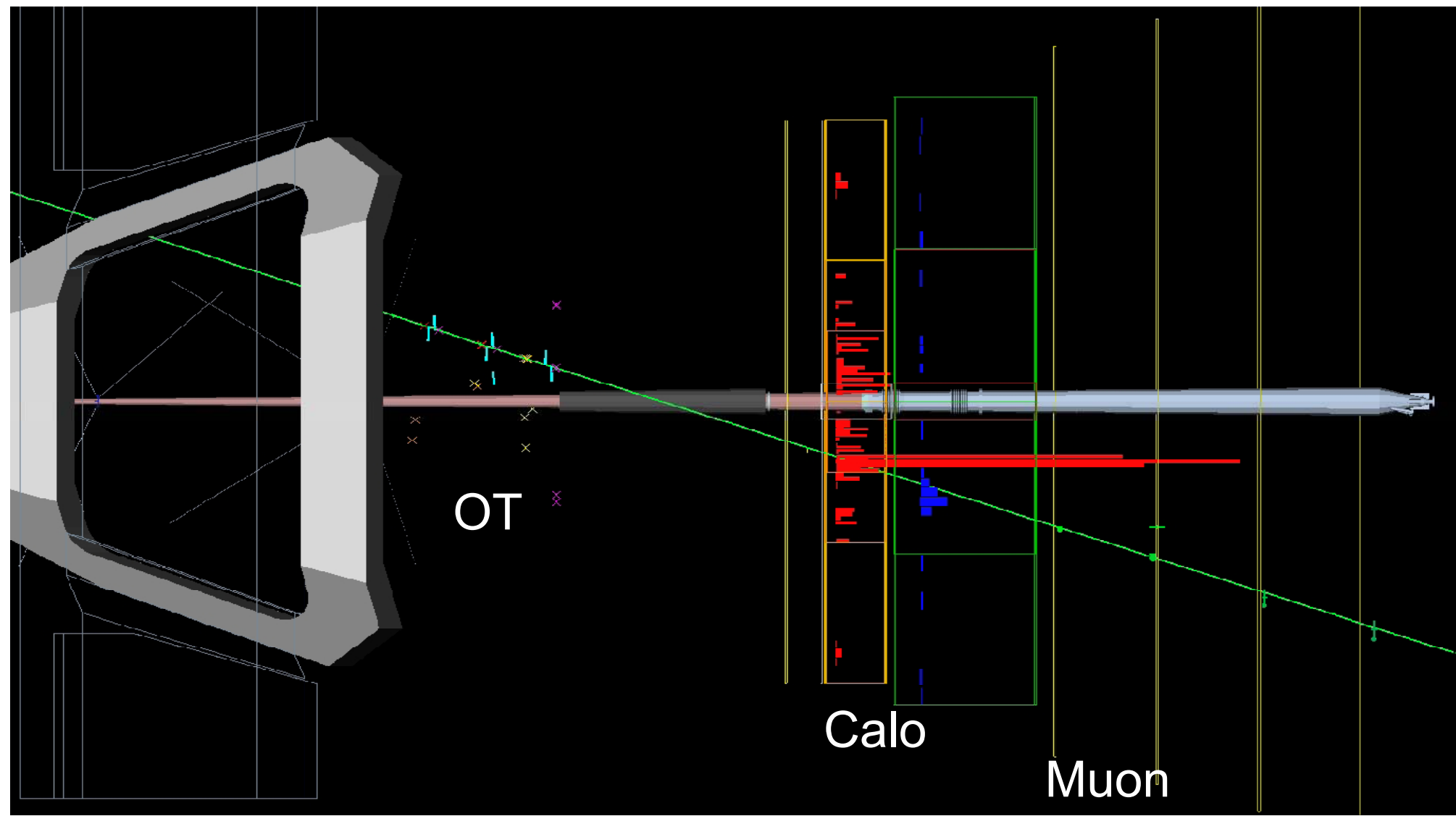




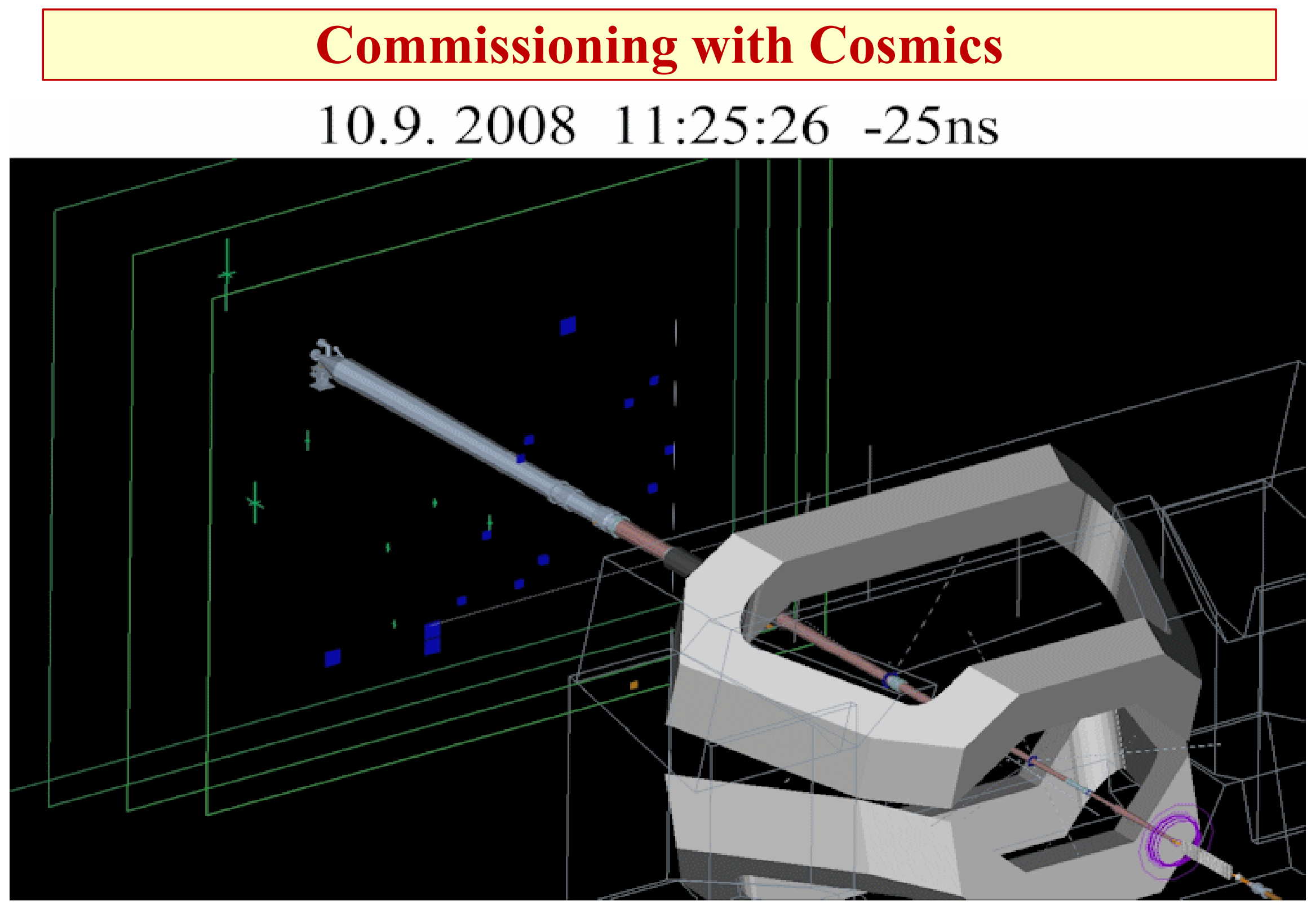

Readout of consecutive $25 \mathrm{~ns}$ crossings for a single trigger LHCP Tsukuba, March 2009 TIPP09 


\section{Conculsions}

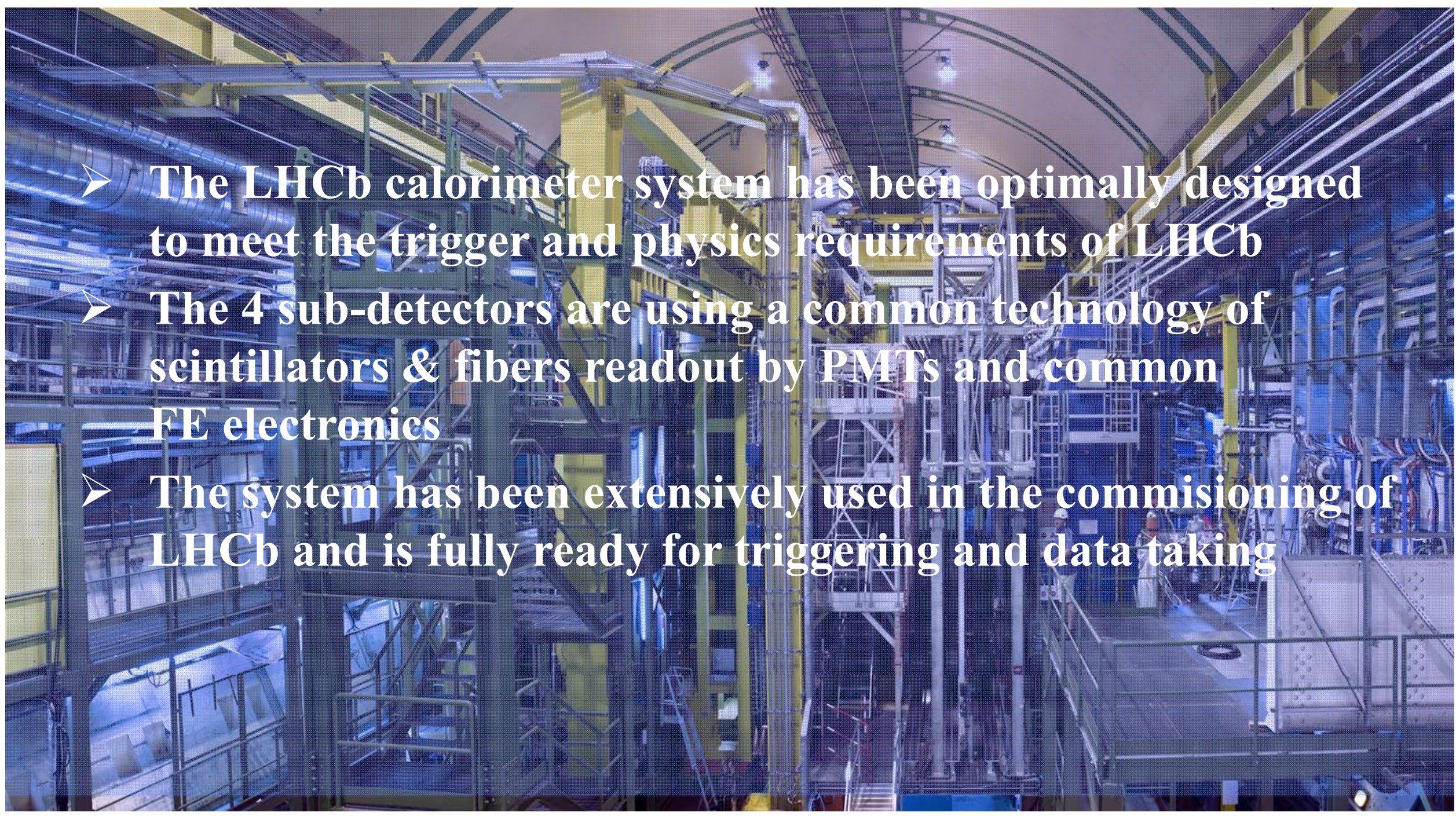

LHCP Tsukuba, March 2009 


\section{Spare slides}




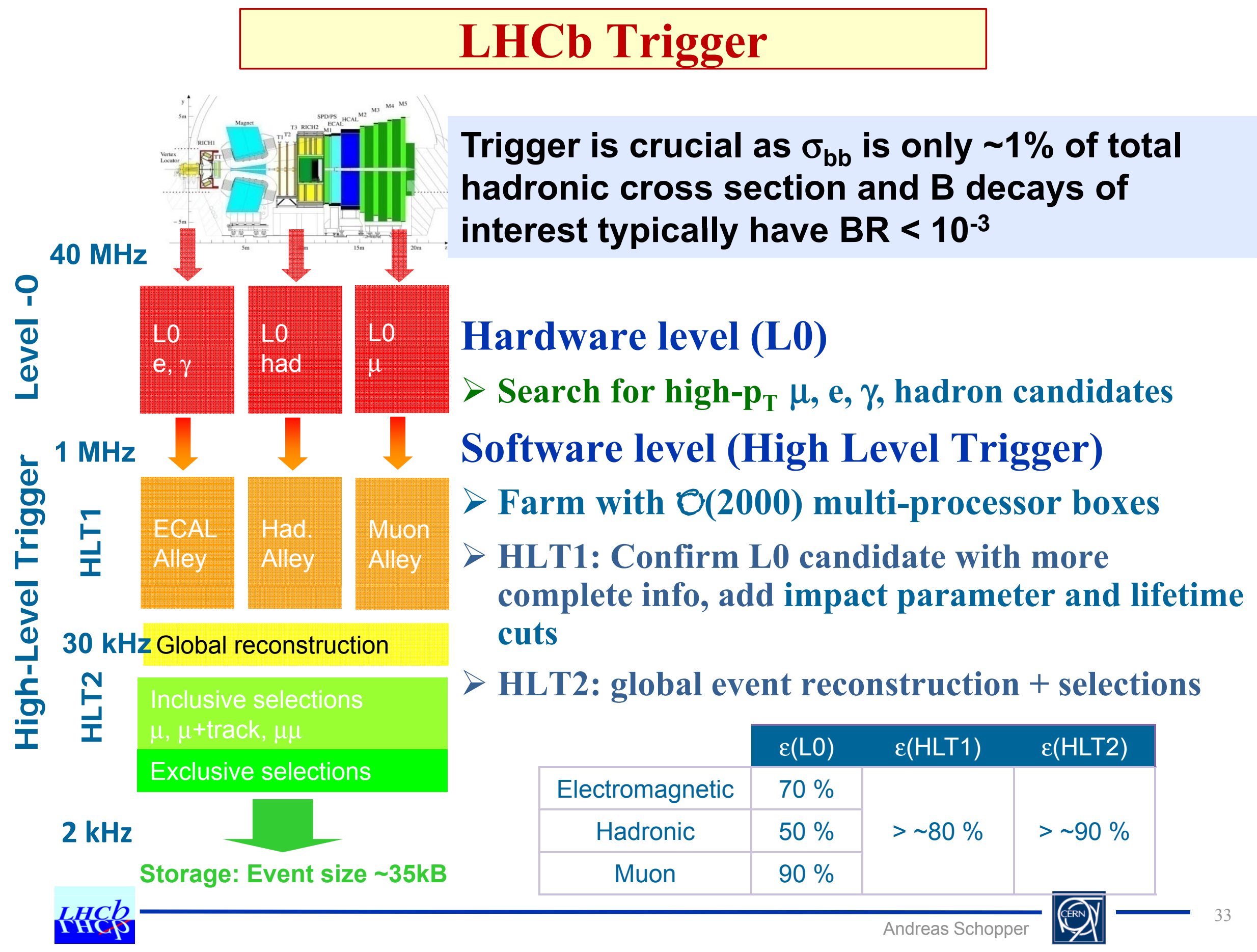




\section{Expected radiation environment}

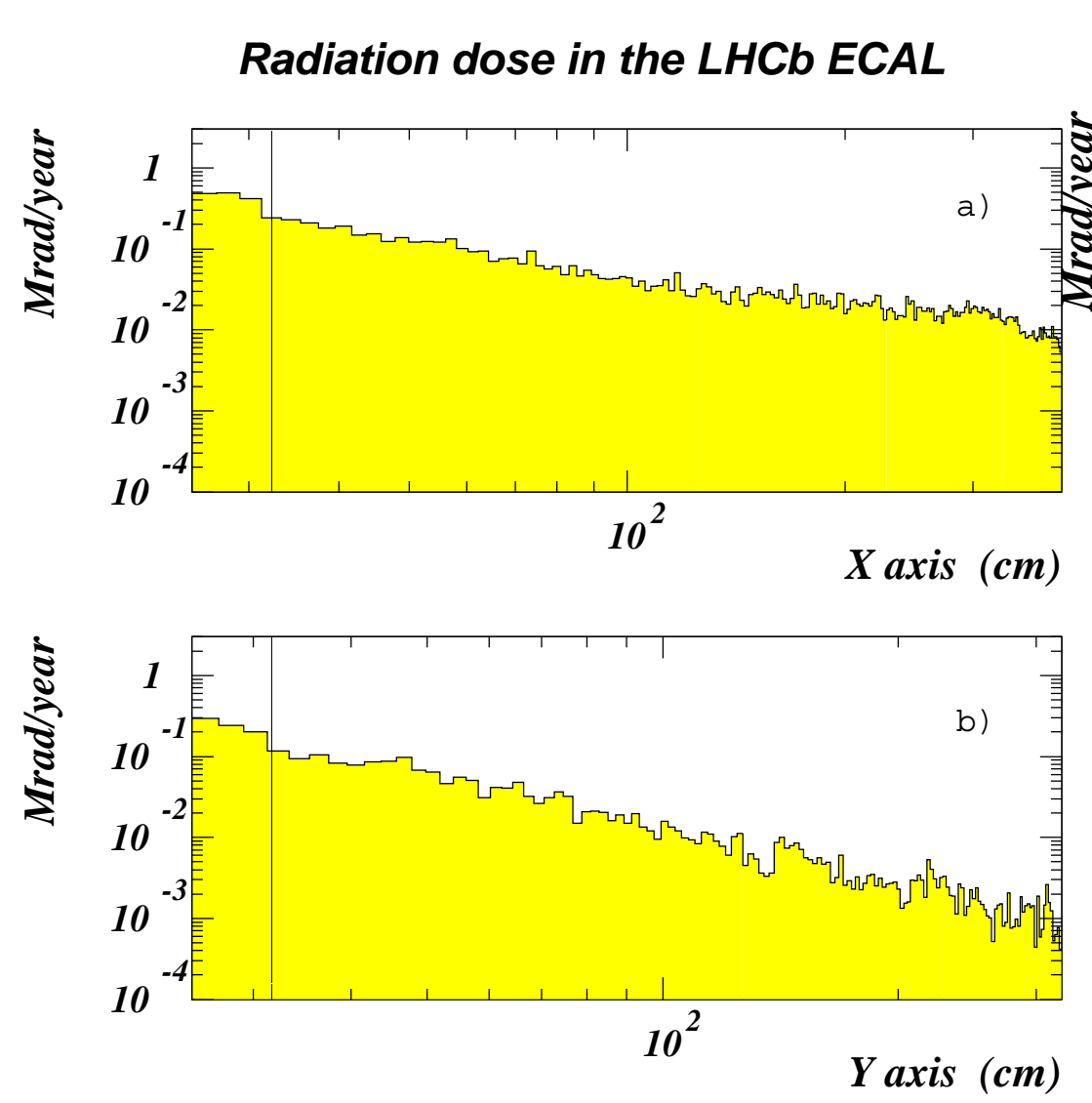

Longitudinal dose in the $L H C b$ ECAL

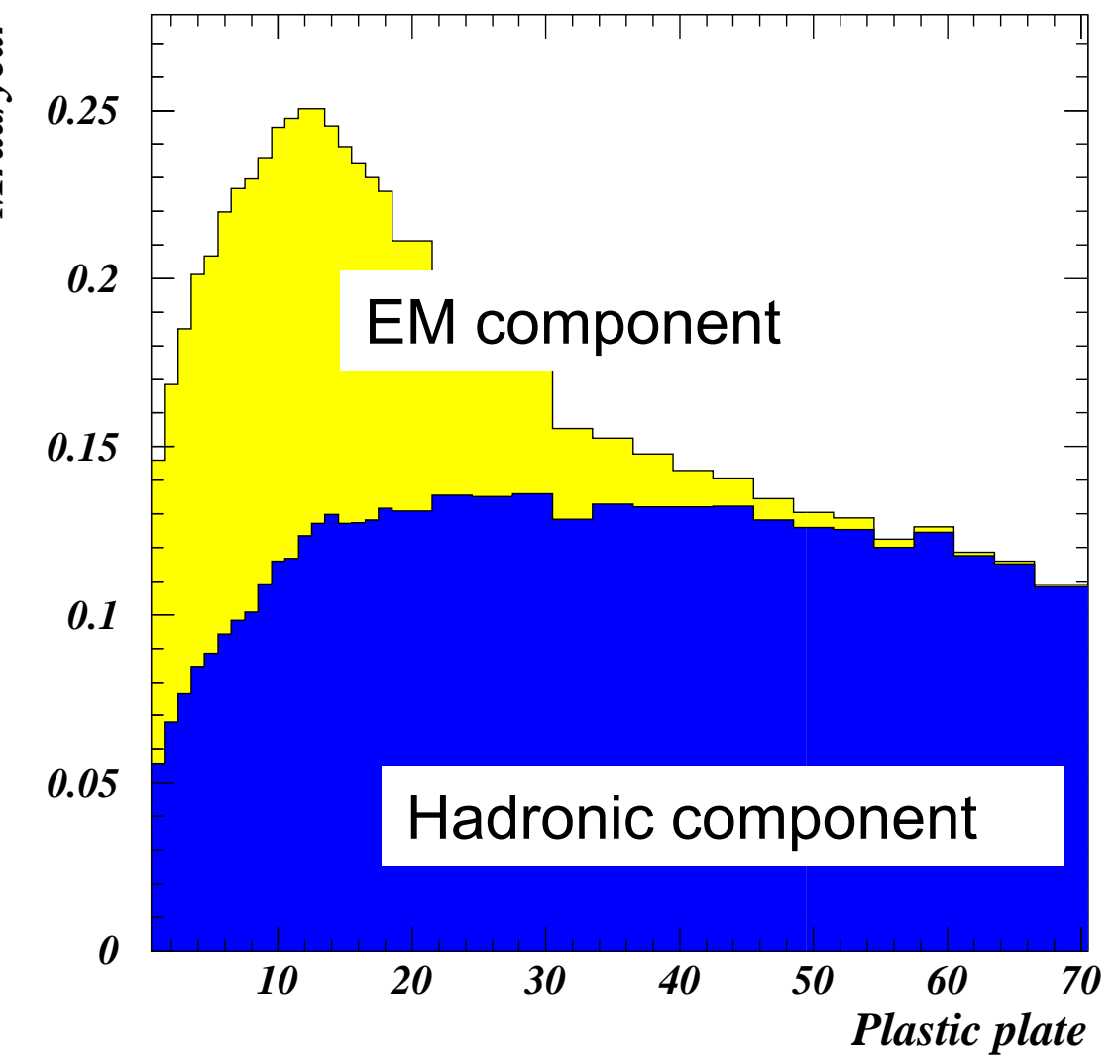




\section{Behavior of module components under irradiation}

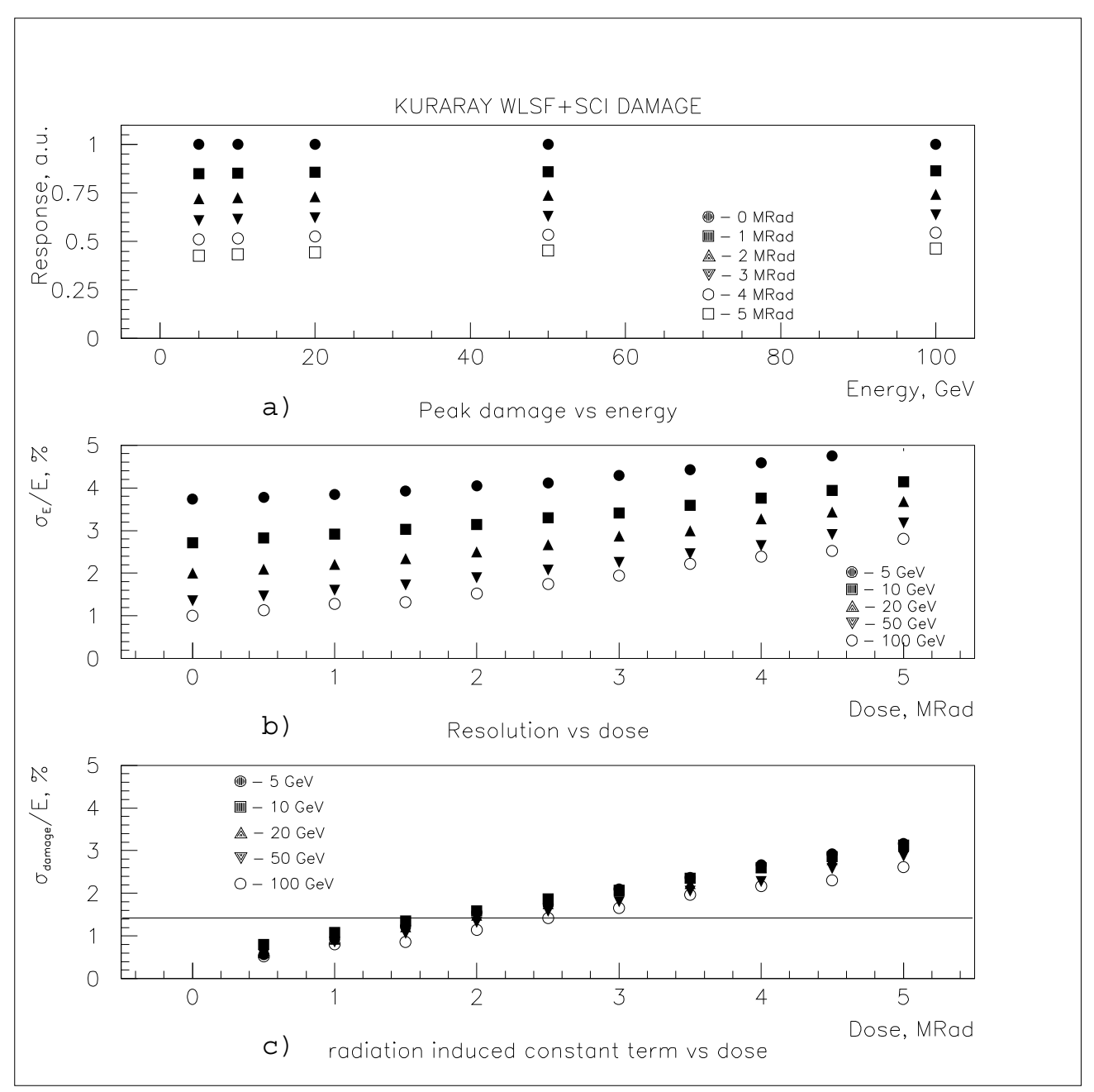

